\title{
Wilson loop form factors: a new duality
}

\author{
Dmitry Chicherin, ${ }^{a}$ Paul Heslop, ${ }^{b}$ Gregory P. Korchemsky ${ }^{c}$ and Emery Sokatchev ${ }^{d, e}$ \\ a PRISMA Cluster of Excellence, Johannes Gutenberg University, \\ 55099 Mainz, Germany \\ ${ }^{b}$ Mathematics Department, Durham University, Science Laboratories, \\ South Rd, Durham DH1 3LE, U.K. \\ ${ }^{c}$ Institut de Physique Théorique, ${ }^{1}$ CEA Saclay, \\ 91191 Gif-sur-Yvette Cedex, France \\ ${ }^{d}$ LAPTh, ${ }^{2}$ Université de Savoie, CNRS, \\ B.P. 110, F-74941 Annecy-le-Vieux, France \\ e Theoretical Physics Department, CERN, \\ CH-1211, Geneva 23, Switzerland \\ E-mail: chicherin@uni-mainz.de, paul.heslop@durham.ac.uk, \\ gregory.korchemsky@ipht.fr, emeri.sokatchev@cern.ch
}

ABSTRACT: We find a new duality for form factors of lightlike Wilson loops in planar $\mathcal{N}=4$ super-Yang-Mills theory. The duality maps a form factor involving an $n$-sided lightlike polygonal super-Wilson loop together with $m$ external on-shell states, to the same type of object but with the edges of the Wilson loop and the external states swapping roles. This relation can essentially be seen graphically in Lorentz harmonic chiral (LHC) superspace where it is equivalent to planar graph duality. However there are some crucial subtleties with the cancellation of spurious poles due to gauge fixing. They are resolved by finding the correct formulation of the Wilson loop and by careful analytic continuation from Minkowski to Euclidean space. We illustrate all of these subtleties explicitly in the simplest non-trivial NMHV-like case.

Keywords: Duality in Gauge Field Theories, Scattering Amplitudes, Superspaces, Wilson, 't Hooft and Polyakov loops

ARXIV EPRINT: 1612.05197

\footnotetext{
${ }^{1}$ Unité de Recherche Associée au CNRS UMR 3681.

${ }^{2}$ Laboratoire d'Annecy-le-Vieux de Physique Théorique, UMR 5108.
} 


\section{Contents}

1 Introduction 2

2 Definitions and summary of the results 4

2.1 Generalised form factors of Wilson loops 4

2.2 Dual variables 5

2.3 Duality relation 6

$\begin{array}{lll}2.4 & \text { Duality relation at MHV level } & 7\end{array}$

2.5 Duality beyond MHV 9

3 Lightlike Wilson loop in LHC superspace $\quad 11$

$3.1 \mathcal{N}=4$ super-Yang-Mills in LHC superspace $\quad 11$

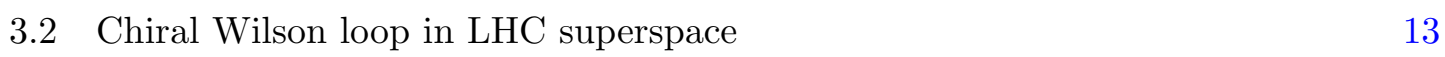

4 Diagrammatic approach to the duality 13

$\begin{array}{lll}4.1 & \text { MHV example } & 14\end{array}$

$\begin{array}{lll}4.2 & \text { Classification of diagrams } & 15\end{array}$

5 Duality between NMHV $\times$ MHV and MHV $\times$ NMHV 16

$\begin{array}{lll}5.1 \text { Cusp diagrams } & 17\end{array}$

$\begin{array}{lll}5.2 & \text { Edge diagrams } & 18\end{array}$

$\begin{array}{lll}5.3 & \text { Spurious pole cancellation } & 19\end{array}$

$\begin{array}{lll}5.4 & \text { Summary of the duality mechanism } & 22\end{array}$

6 Concluding remarks $\quad 23$

A Chiral lightlike Wilson loop in LHC superspace $\quad 25$

$\begin{array}{lll}\text { A.1 Chiral lightlike Wilson loop } & 25\end{array}$

A.2 Bridge transformation to LHC superspace 26

A.3 Comments on the twistor formulation of Mason and Skinner 28

$\begin{array}{ll}\text { B Feynman rules } & 28\end{array}$

B.1 Propagators and on-shell states 28

B.2 Effective Feynman rules 30

$\begin{array}{lll}\text { B.2.1 Vertices } & 30\end{array}$

$\begin{array}{lll}\text { B.2.2 Propagators } & 30\end{array}$

$\begin{array}{ll}\text { C Fourier transforms } & 31\end{array}$

D Cancellation of spurious poles: boundary cases 32 


\section{Introduction}

The natural gauge invariant objects in any gauge theory include scattering amplitudes, Wilson loops, correlation functions and form factors of local operators. In the past years numerous studies have revealed interesting duality relations between the first three objects in planar $\mathcal{N}=4$ SYM theory. The simplest MHV gluon scattering amplitude $\mathrm{A}_{n}\left(p_{1}, \ldots, p_{n}\right)$ has been shown [1-3] to be dual to a Wilson loop $\mathcal{W}_{n}\left(x_{1}, \ldots, x_{n}\right)$ defined on a lightlike contour,

$$
\mathrm{A}_{n}\left(p_{1}, \ldots, p_{n}\right)=\mathcal{W}_{n}\left(x_{1}, \ldots, x_{n}\right),
$$

upon the identification of the separation between the cusp points $x_{i}$ of the contour with the particle momenta $p_{i}$ in Minkowski space, $x_{i}-x_{i+1}=p_{i}$ for $i=1, \ldots, n$ and $x_{n+1} \equiv x_{1}$. This duality has a natural supersymmetric extension [4-6] where the super-lightlike contour is built out of the on-shell supermomenta of the scattered particles. The correlation functions $G_{n}=\left\langle O\left(x_{1}\right) \ldots O\left(x_{n}\right)\right\rangle$ of local gauge invariant operators $O(x)$ are dual to the Wilson loops (and hence to the amplitudes) in the lightlike limit [7, 8], $\lim _{x_{i, i+1}^{2} \rightarrow 0} x_{12}^{2} \ldots x_{n 1}^{2} G_{n}=\mathcal{W}_{n}$. This duality has a supersymmetric generalisation as well [9-11].

The fourth object is the form factor $\left\langle 0|O(x)| k_{1}, \ldots, k_{m}\right\rangle$ of a local operator $O(x)$ with an asymptotic $m$-particle state of on-shell momenta $k_{j}^{2}=0$ for $j=1, \ldots, m$. It is a hybrid between correlation functions and scattering amplitudes because it lives simultaneously in coordinate and momentum spaces. Such form factors (and their supersymmetric extensions in $\mathcal{N}=4 \mathrm{SYM}$ ) have been actively studied in recent years [12-18]. It is interesting to know if there are possible duality relations for them as well. This question has been addressed in [19] but for a more complicated object, the matrix element of a lightlike bosonic Wilson loop stretched between local operators along a single light-cone direction, with an on-shell state. It has been shown that this object is dual to itself upon swapping the coordinate and momentum data. It has also been conjectured there that the new duality may extend to a larger class of objects, namely the form factor $W_{n, m}=\left\langle 0\left|\mathcal{W}_{n}\left(x_{1}, \ldots, x_{n}\right)\right| k_{1}, \ldots, k_{m}\right\rangle$ of an $n$-gon lightlike (supersymmetric) Wilson loop with an $m$-particle state. Schematically, the suggested duality takes the form

$$
W_{n, m}(\{x\} \mid\{k\})=W_{m, n}(\{y\} \mid\{p\}),
$$

where the kinematical data on both sides are related as in (1.1),

$$
x_{i}-x_{i+1}=p_{i}, \quad y_{j}-y_{j+1}=k_{j},
$$

for $i=1, \ldots, n$ and $j=1, \ldots, m$ provided that the total momenta of the particles vanish, $\sum_{i=1}^{n} p_{i}=\sum_{j=1}^{m} k_{j}=0$. This conjecture has been successfully tested in [20] in the simplest case of a Wilson loop with a state of helicity $(+1)$ gluons and in the Born approximation.

Building upon the observations in [19] and [20], in this paper we study the general case of the form factor for a lightlike supersymmetric Wilson loop and we argue that it has a remarkable duality property in planar $\mathcal{N}=4 \mathrm{SYM}$. It extends the bosonic relation (1.2) and the identification of coordinates with momenta (1.3) to their supersymmetric analogs. The super-Wilson loop form factors are considered in the planar limit and in the lowestorder perturbative approximation (Born level). The introduction of Grassmann variables 
( $\theta_{i}$ on the Wilson loop contour and $\eta_{j}$ for the on-shell states) allows us to probe the duality for more complicated configurations of particle helicities. By analogy with the amplitudes, we call the contributions at the lowest level in the Grassmann expansion MHV-like, at the next level NMHV-like, etc. At MHV level we confirm the result of [20]. The NMHV level is much more complicated, the form factor being a non-trivial rational function of the kinematical data. Yet, we show that the duality still works, in a rather simple and suggestive way, by just matching planar Feynman diagrams. This allows us to argue that it should hold for the complete supersymmetric object (at all Grassmann levels) and also beyond the Born approximation.

The key to understanding the duality is the appropriate superspace formulation of the Wilson loop and its form factor. In the conventional approach the chiral supersymmetric Wilson loop [4-6] is formulated in terms of constrained on-shell super-connections [21, 22], which makes the Feynman diagram technique highly inefficient. In this paper we prefer to use the Lorentz harmonic chiral (LHC) superspace approach [23]. It provides an offshell formulation of the chiral $\mathcal{N}=4 \mathrm{SYM}$ theory in terms of unconstrained prepotentials, best suited for supersymmetric quantisation. LHC superspace is an alternative to the twistor formulation [24, 25], closer in spirit to traditional field theory (see also [26]). The main idea is to consider the interacting theory as a perturbation of the self-dual sector. The twistor formulation has been successfully used to justify the so-called MHV rules for the computation of the amplitude [27], to prove the duality between supersymmetric Wilson loops and amplitudes [5], to compute off-shell correlation functions of the $\mathcal{N}=4$ stress-tensor multiplet [28]. More recently, the LHC formalism was applied to finding the non-chiral completion of the correlators [29] and to the calculation of form factors of local operators [30]. In this paper, after explaining the kinematical setup in section 2, we formulate the lightlike Wilson loop in LHC superspace in section 3 and apply the Feynman rules of [30] to the computation of its form factors in section 4 . We find an important additional contribution to the Wilson loop, compared to the twistor formulation [5]. It is needed to make the Wilson loop gauge invariant.

The duality essentially works on a graph-to-graph basis. More precisely, we find two types of Feynman graphs corresponding to two different helicity configurations at NMHVlike level. These graphs are dual to each other after identifying the kinematical data as in (1.3) and redrawing the graph following a simple rule. In addition to these graphs there are sets of graphs whose role is to restore gauge invariance. We use a light-cone gauge whose parameter is the so-called reference spinor. A known problem of such gauges is the presence of spurious poles. Their elimination in the Feynman graphs (and hence the restoration of gauge invariance) is a somewhat subtle procedure which we describe in detail in section 5 .

We end the paper with several appendices. In appendix A we explain how to obtain the LHC formulation of the lightlike Wilson loop starting from the standard one with constrained super-connections. In appendix B we summarise the Feynman rules in the lightcone gauge. In appendix $\mathrm{C}$ we derive some Fourier transforms that we need for establishing the duality. In appendix D we explain the mechanism of spurious pole cancellation in the boundary cases. 


\section{Definitions and summary of the results}

\subsection{Generalised form factors of Wilson loops}

In this paper, we study a new object - the generalised form factor of the lightlike Wilson loop. In $\mathcal{N}=4 \mathrm{SYM}$ with gauge group $\mathrm{SU}(N)$ it is defined as the matrix element of a lightlike $n$-gon supersymmetric Wilson loop $\mathcal{W}_{n}$ with the on-shell $m$-particle state $\left|1^{a_{1}} \ldots m^{a_{m}}\right\rangle$ :

$$
\left\langle 0\left|\mathcal{W}_{n}\right| 1^{a_{1}} \ldots m^{a_{m}}\right\rangle=\frac{1}{N}\left\langle 0\left|\operatorname{tr} P \exp \left[i \oint_{\mathcal{C}_{n}}\left(d x^{\mu} \mathcal{A}_{\mu}(x, \theta)+d \theta^{\alpha A} \mathcal{A}_{\alpha A}(x, \theta)\right)\right]\right| 1^{a_{1}} \ldots m^{a_{m}}\right\rangle,
$$

where the integration goes over a closed contour $\mathcal{C}_{n}$ formed by $n$ straight lightlike segments connecting the superspace points $\left(x_{i}, \theta_{i}\right)$. The bosonic and fermionic gauge connections, $\mathcal{A}_{\mu}$ and $\mathcal{A}_{\alpha A}$, have expansions in powers of $\theta$ 's with coefficients given in terms of the gluon, gaugino and scalar fields. Their explicit expressions are shown below in (2.28).

In the planar limit, the form factor can be decomposed in the standard manner over the basis of single traces,

$$
\left\langle 0\left|\mathcal{W}_{n}\right| 1^{a_{1}} \ldots m^{a_{m}}\right\rangle=\sum_{\sigma \in S_{m} / Z_{m}} \operatorname{tr}\left(T^{a_{\sigma_{1}}} \ldots T^{a_{\sigma_{m}}}\right) F_{n, m}\left(\sigma_{1}, \ldots, \sigma_{m}\right),
$$

where the sum runs over all permutations of the external particles $\sigma_{1}, \ldots, \sigma_{m}$ modulo cyclic shifts. The matrix element (2.2) is a natural generalisation of lightlike Wilson loops $\left\langle 0\left|\mathcal{W}_{n}\right| 0\right\rangle$ and scattering amplitudes $\mathrm{A}\left(1^{a_{1}} \ldots m^{a_{m}}\right)$. In fact, it gets a disconnected contribution given by their product. In what follows we discard it and consider only the connected contribution to $(2.2)$.

The color-ordered form factors $F_{n, m}$ depend on two sets of variables. The first set consists of $n$ coordinates in Minkowski space-time and their odd superpartners $\left(x_{i}^{\dot{\alpha} \alpha}, \theta_{i}^{\alpha A}\right)$ specifying the position of the vertices of a lightlike $n$-gon, ${ }^{1}$

$$
\left(x_{i}-x_{i+1}\right)^{2}=0, \quad\left(x_{i}-x_{i+1}\right)^{\dot{\alpha} \alpha}\left(\theta_{i, \alpha}^{A}-\theta_{i+1, \alpha}^{A}\right)=0
$$

for $i=1, \ldots, n$, with the cyclicity conditions $x_{n+1}=x_{1}$ and $\theta_{n+1}=\theta_{1}$. Here the first relation means that the Wilson loop is built from lightlike segments and the second relation is its superpartner.

The second set of variables consists of the on-shell momenta of $m$ particles $\left(k_{j}^{\dot{\alpha} \alpha}, \eta_{j A}\right)$

$$
\left.k_{j}^{\dot{\alpha} \alpha}=\tilde{k}_{j}^{\dot{\alpha}} k_{j}^{\alpha} \equiv \mid k_{j}\right]\left\langle k_{j}\right|
$$

with $k_{j}^{2}=0$ and $j=1, \ldots, m$. Like the scattering amplitudes, the expansion of the on-shell state in powers of $\eta_{j A}$ corresponds to particles with different helicity (gluons, gaugini and scalars). Each particle superstate carries one unit of helicity. It is then convenient to introduce the helicity-free function $W_{n, m}$ multiplying (2.2) by the so-called Parke-Taylor factor

$$
W_{n, m}=\left\langle k_{1} k_{2}\right\rangle\left\langle k_{2} k_{3}\right\rangle \ldots\left\langle k_{m} k_{1}\right\rangle F_{n, m}(1, \ldots, m),
$$

\footnotetext{
${ }^{1}$ We use two-component spinor notation for vectors, e.g., $x^{\dot{\alpha} \alpha}=\left(\sigma_{\mu}\right)^{\dot{\alpha} \alpha} x^{\mu}$. The Lorentz and R symmetry indices take values $\alpha=1,2, \dot{\alpha}=1,2$ and $A=1,2,3,4$, respectively.
} 
where $\left\langle k_{i} k_{j}\right\rangle=k_{i}^{\alpha} \epsilon_{\alpha \beta} k_{j}^{\beta}$. The scalar function $W_{n, m}$ defined in this way depends on the two sets of variables introduced above,

$$
W_{n, m}=W_{n, m}(\{x, \theta\} ;\{k, \eta\}) .
$$

As follows from the definition (2.2), this function is invariant under cyclic shifts of the coordinates and momenta.

\subsection{Dual variables}

To elucidate the interesting properties of $W_{n, m}$ we introduce the so-called dual superspace variables [31]. The coordinates of the Wilson loop $\left(x_{i}, \theta_{i}^{A}\right)$ have the dual momenta $\left(p_{i}, \omega_{i}^{A}\right)$ defined as

$$
x_{i}-x_{i+1}=p_{i}, \quad\left|\theta_{i}^{A}\right\rangle-\left|\theta_{i+1}^{A}\right\rangle=\left|p_{i}\right\rangle \omega_{i}^{A},
$$

where we do not display the Lorentz indices for simplicity. It follows from (2.3) that $p_{i}$ are lightlike vectors, $p_{i}^{2}=0$, satisfying the condition $\sum_{i=1}^{n} p_{i}=0$. Similarly, the odd variables $\omega_{i}^{A}$ satisfy the relation $\sum_{i=1}^{n}\left|p_{i}\right\rangle \omega_{i}^{A}=0$ and solve the second condition in (2.3). Note that the properties of $\left(p_{i}, \omega_{i}^{A}\right)$ (with $\left.i=1, \ldots, n\right)$ match those of the supermomenta of the on-shell states in the scattering amplitude $\mathrm{A}_{n}$. This observation was crucial in establishing the duality between the lightlike Wilson loop $\mathcal{W}_{n}$ and the scattering amplitude $\mathrm{A}_{n}$.

For the set of on-shell momenta $\left(k_{j}, \eta_{j A}\right)$, the dual coordinates are defined as

$$
k_{j}=y_{j}-y_{j+1}, \quad\left|k_{j}\right\rangle \eta_{j A}=\left|\psi_{j, A}\right\rangle-\left|\psi_{j+1, A}\right\rangle .
$$

Here the dual momenta $y_{1}, \ldots, y_{m+1}$ are consecutively lightlike separated, $\left(y_{i}-y_{i+1}\right)^{2}=$ 0 and their superpartners satisfy $\left(y_{j}-y_{j+1}\right)\left(\left|\psi_{j, A}\right\rangle-\left|\psi_{j+1, A}\right\rangle\right)=0$. Note the striking similarity between relations (2.7) and (2.8). Namely, these relations can be mapped into each other by exchanging coordinates with dual momenta, $(x, \theta) \rightarrow(y, \psi)$, and momenta with dual coordinates, $(k, \eta) \rightarrow(p, \omega)$.

There is however an important difference between the two sets of dual coordinates. The dual vectors $p_{i}$ define the edges of a closed $n$-gon and their sum equals zero. The same is true for the sum of dual odd coordinates $\left|p_{i}\right\rangle \omega_{i}^{A}$,

$$
\sum_{i=1}^{n} p_{i}=x_{1}-x_{n+1}=0, \quad \sum_{i=1}^{n}\left|p_{i}\right\rangle \omega_{i}^{A}=\left|\theta_{1}^{A}\right\rangle-\left|\theta_{n+1}^{A}\right\rangle=0,
$$

so that the dual variables satisfy the periodicity conditions $x_{i}=x_{i+n}$ and $\theta_{i}^{A}=\theta_{i+n}^{A}$. For the dual momenta the analogous relations read

$$
\sum_{j=1}^{m} k_{j}=y_{1}-y_{m+1}=K, \quad \sum_{j=1}^{m}\left|k_{j}\right\rangle \eta_{j A}=\left|\psi_{1, A}\right\rangle-\left|\psi_{m+1, A}\right\rangle=Q_{A},
$$

where $K$ and $Q$ are the total momentum and supercharge of the $m$ particles in (2.2), respectively. In contrast with (2.9), $K$ and $Q$ can take arbitrary values and there are no reasons to impose the periodicity conditions $y_{m+1}=y_{1}$ and $\psi_{m+1, A}=\psi_{1, A}$. Indeed, the function (2.6) is well defined for arbitrary $K$ and $Q$. 

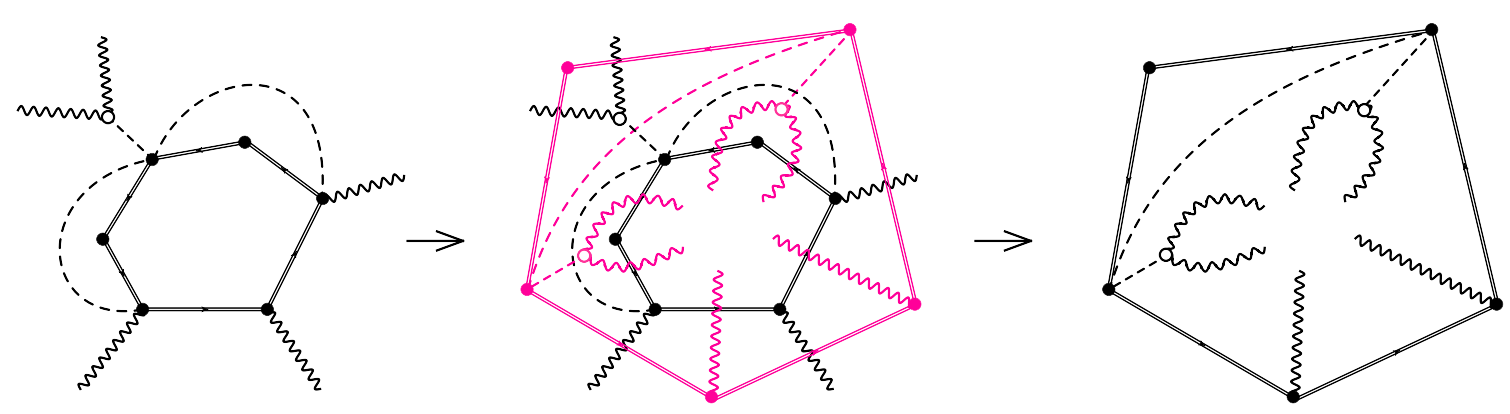

Figure 1. Diagrammatic representation of the duality relation (2.13). The Wilson loop on the left is built out of lightlike vectors $p_{1}, \ldots, p_{n}$, the wavy lines denote on-shell particles with momenta $k_{1}, \ldots, k_{m}$ and the dash lines stand for free propagators. Black and white dots denote effective vertices. The dual Wilson loop form factor on the right has the lightlike vectors and momenta exchanged. The middle figure explains the duality by superimposing the two graphs.

\subsection{Duality relation}

Setting $K=Q_{A}=0$ in (2.10) we restore the symmetry between (2.9) and (2.10). This allows us to treat the original variables and their dual counterparts on an equal footing. In this paper we argue that for $K=Q_{A}=0$ the symmetry of $W_{n, m}$ is enhanced and yields an interesting duality relation for $W_{n, m}$ that we shall formulate in a moment. More precisely, we can use the dual variables to define, following (2.2), the matrix element of the lightlike Wilson loop $\left\langle 0\left|\mathcal{W}_{m}\right| 1^{a_{1}} \ldots n^{a_{n}}\right\rangle$. Here the Wilson loop is evaluated along a closed lightlike $m$-gon with vertices located at $\left(y_{j}, \psi_{j}\right)$ and the on-shell state consists of $n$ particles with supermomenta $\left(p_{i}, w_{i}^{A}\right)$. This matrix element has the same general form (2.2) and (2.5), with the corresponding scalar function $W_{m, n}$ given by

$$
W_{m, n}=W_{m, n}(\{y, \psi\} ;\{p, \omega\}) .
$$

Applying relations (2.7) and (2.8) we can express it in terms of the original variables $\left\{x_{i}, \theta_{i}\right\}$ and $\left\{k_{j}, \eta_{j}\right\}$.

The duality relation that we propose states that the functions (2.6) and (2.11) coincide in planar $\mathcal{N}=4 \mathrm{SYM}$,

$$
W_{n, m}(\{x, \theta\} ;\{k, \eta\})=W_{m, n}(\{y, \psi\} ;\{p, \omega\}) .
$$

Using the definition of the dual variables we can rewrite the duality relation in other equivalent forms, e.g.

$$
W_{n, m}(\{p, \omega\} ;\{k, \eta\})=W_{m, n}(\{k, \eta\} ;\{p, \omega\}) .
$$

This relation is represented diagrammatically in figure 1.

The duality relation (2.12) should hold for any values of $n$ and $m$. As a simple illustration, we examine it for the lowest values of $n$ and $m$. In the special cases $m=0$ (or $n=0$ ) we recover the well-known duality between the $n$-point superamplitude and the $n$-point super-Wilson loop. Since the $n$-gon Wilson loop is well defined for $n \geq 2$, 
we start with $n=2,3$. In this case, the cusp points $x_{i}$ satisfying (2.3) have to lie on the same light-ray in Minkowski space-time. Then, the integration contour of the Wilson loop collapses to a backtracking path leading to $W_{2}=W_{3}=1$. As a consequence, the matrix element on the left-hand side of (2.2) only receives disconnected contributions yielding the vanishing of $W_{n, m}(\{x, \theta\} ;\{k, \eta\})$ for $n=2,3$. The duality relation (2.12) implies that the same should be true for $W_{m, n}(\{y, \psi\} ;\{p, \omega\})$ for $n=2,3$. Indeed, the corresponding matrix element (2.2) involves an on-shell state with (real valued) lightlike momenta $k_{i}$ that are necessarily aligned due to $\sum_{i} k_{i}=0$. In this case $\left\langle k_{i} k_{j}\right\rangle=0$ and it follows from (2.5) that $W_{m, n}$ vanishes, in agreement with (2.11).

\subsection{Duality relation at MHV level}

Let us now consider the duality relation for $n, m \geq 4$. In this case both sides of (2.12) are different from zero and are given by nontrivial functions of the kinematical variables and of the 't Hooft coupling constant. In what follows we shall restrict our consideration to the lowest order in the coupling (Born level). Expanding both sides of (2.12) in the Grassmann variables, we can get relations between the different components. By analogy with the scattering amplitudes, we shall refer to the terms of the expansion as MHV, NMHV, etc. Notice that since $W_{n, m}(\{x, \theta\} ;\{k, \eta\})$ depends on two sets of Grassmann variables $\theta_{i}$ and $\eta_{j}$, we will have to deal with a double expansion of the form $\mathrm{N}^{\kappa} \mathrm{MHV} \times \mathrm{N}^{\sigma} \mathrm{MHV}$.

The lowest term of the expansion, MHV $\times$ MHV, corresponds to (2.12) with all Grassmann variables put to zero on both sides of the relation. Namely, for $\theta_{i}=0$ the super Wilson loop $\mathcal{W}_{n}$ reduces to the bosonic lightlike Wilson loop and for $\eta_{j}=0$ the on-shell state in (2.2) reduces to a gluon state of helicity $(+1)$. In this way, from (2.1) and (2.2) we obtain

$$
F_{n, m}^{\mathrm{MHV} \times \mathrm{MHV}}(x, k)=\frac{1}{N}\left\langle 0\left|\operatorname{tr}\left(E_{1 n} \ldots E_{32} E_{21}\right)\right| k_{1}^{+} \ldots k_{m}^{+}\right\rangle,
$$

where $E_{i+1, i}$ denotes a bosonic Wilson line in the fundamental of $\mathrm{SU}(N)$ evaluated along the lightlike segment $\left[x_{i}, x_{i+1}\right]$

$$
E_{i+1, i}=P \exp \left(-i \int_{0}^{1} d t p_{i} \cdot A\left(x_{i}-p_{i} t\right)\right)
$$

with $p_{i}=x_{i}-x_{i+1}$. Notice that the ordering of the $E$-factors inside the trace in (2.14) is opposite to that of the gluons in the on-shell state.

In the Born approximation, $\mathrm{A}_{n, m}^{\mathrm{MHV} \times \mathrm{MHV}}$ is given by the sum of tree Feynman diagrams in which the on-shell gluons are attached to the lightlike $n$-gon contour either directly or through $3-$ and 4-gluon interaction vertices. The calculation can be simplified by introducing the notion of a "wedge", i.e. a cusped Wilson line built from two semi-infinite rays running along the lightlike vectors $-p_{1}$ and $p_{2}$ and joining at point $x$ :

$$
W_{p_{2}, p_{1}}(x)=P\left[\exp \left(i \int_{0}^{\infty} d t p_{2} \cdot A\left(x+p_{2} t\right)\right) \exp \left(-i \int_{-\infty}^{0} d t p_{1} \cdot A\left(x-p_{1} t\right)\right)\right] .
$$

In the product $W_{p_{3}, p_{2}}\left(x_{3}\right) W_{p_{2}, p_{1}}\left(x_{2}\right)$ with $p_{2}=x_{2}-x_{3}$, it is easy to see that the two semiinfinite rays running along $p_{2}$ partially cancel against each other giving rise to $E_{32}$. In this 

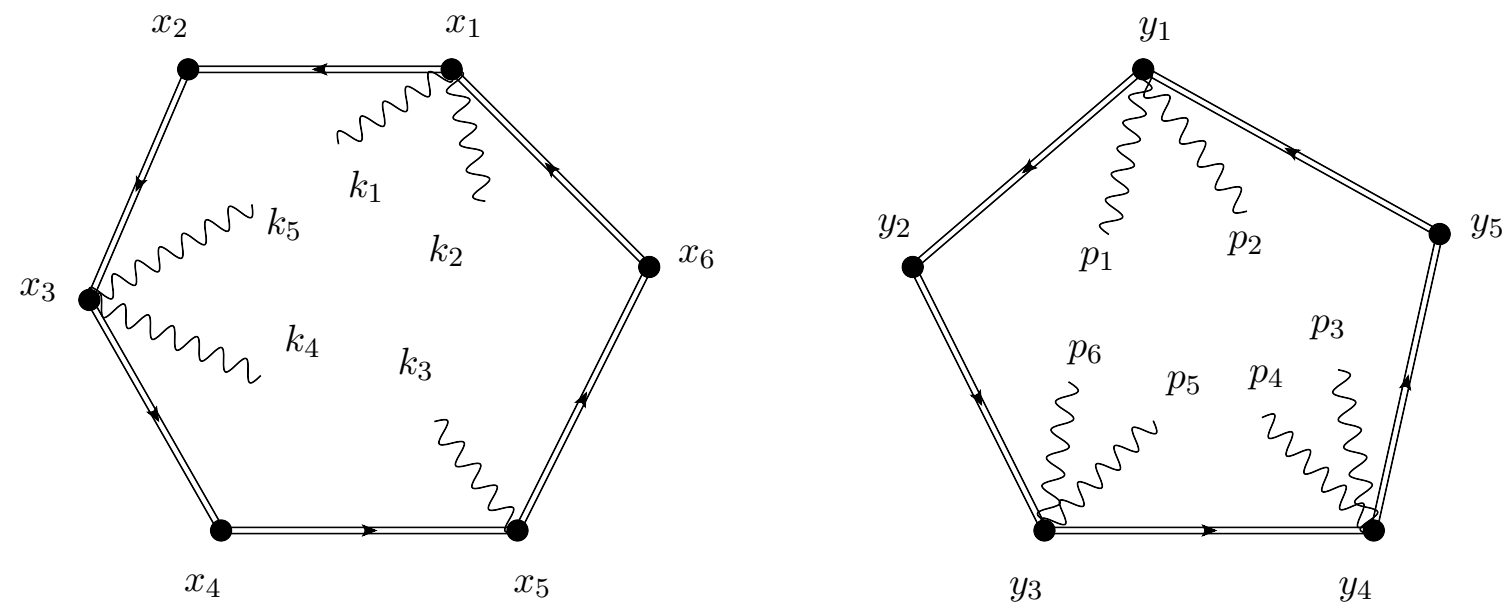

Figure 2. Diagrammatic representation of the duality relation (2.25) for $n=6$ and $m=5$. Notice that the polygon vertices and the gluons are ordered in opposite directions. Black blobs with outgoing gluons denote wedge form factors (2.19). The lightlike edges of the Wilson loops are mapped to the momenta of the on-shell gluons, $k_{i}=y_{i}-y_{i+1}$ and $p_{j}=x_{j}-x_{j+1}$.

way, we can rewrite $(2.14)$ as

$$
F_{n, m}^{\mathrm{MHV} \times \operatorname{MHV}}(x, k)=\frac{1}{N}\left\langle 0\left|\operatorname{tr}\left[W_{p_{n}, p_{n-1}}\left(x_{n}\right) \ldots W_{p_{2}, p_{1}}\left(x_{2}\right) W_{p_{1}, p_{n}}\left(x_{1}\right)\right]\right| k_{1}^{+} \ldots k_{m}^{+}\right\rangle .
$$

The advantage of this representation is that, in the Born approximation, the on-shell gluons can be emitted by one of the $W$-factors thus allowing us to express the matrix element on the right-hand side of (2.17) as the sum over all possible attachments of $m$ gluons to $n$ wedges

$$
\begin{aligned}
F_{n, m}^{\mathrm{MHV} \times \mathrm{MHV}}= & \sum_{\ell_{1}<\cdots<\ell_{s}} \sum_{1 \leq i_{s}<\cdots<i_{1} \leq n}\left\langle 0\left|W_{p_{i_{1}}, p_{i_{1}-1}}\left(x_{i_{1}}\right)\right| k_{\ell_{1}}^{+} \ldots k_{\ell_{2}-1}^{+}\right\rangle \\
& \times\left\langle 0\left|W_{p_{i_{2}}, p_{i_{2}-1}}\left(x_{i_{2}}\right)\right| k_{\ell_{2}}^{+} \ldots k_{\ell_{3}-1}^{+}\right\rangle \ldots\left\langle 0\left|W_{p_{i_{s}}, p_{i_{s}-1}}\left(x_{i_{s}}\right)\right| k_{\ell_{s}}^{+} \ldots k_{\ell_{1}-1}^{+}\right\rangle .
\end{aligned}
$$

Here the first sum goes over all possible partitions of $m$ gluons over $s$ clusters (with $s \leq$ $n$ ) and the second sum runs over all possible wedges $x_{i_{1}}, \ldots, x_{i_{s}}$ to which these clusters are attached. The difference in the ordering of indices $\ell_{k}$ and $i_{k}$ in (2.18) is due to the opposite ordering of the $E$-factors and gluons in (2.14). Relation (2.18) is represented diagrammatically in figure 2 .

Relation (2.18) involves the so-called wedge form factor $\left\langle 0\left|W_{p_{2}, p_{1}}(x)\right| k_{1}^{+} \ldots k_{\ell}^{+}\right\rangle$. Since the on-shell state contains only gluons of the same helicity, its calculation in the Born approximation can be performed in the self-dual sector of Yang-Mills theory [20, 26]

$$
\left\langle 0\left|W_{p_{2}, p_{1}}(x)\right| k_{1}^{+} \ldots k_{\ell}^{+}\right\rangle=F\left(p_{2}, k_{1}, \ldots, k_{\ell}, p_{1}\right) e^{i x\left(k_{1}+\ldots+k_{\ell}\right)} .
$$

Here the dependence on $x$ is fixed by Poincaré symmetry and the order of the arguments of the $F$-function matches the color ordering of the gluons. Its explicit expression reads 
(see section 4.1 for more details)

$$
F\left(p_{2}, k_{1}, \ldots, k_{\ell}, p_{1}\right)=\frac{\left\langle p_{2} p_{1}\right\rangle}{\left\langle p_{2} k_{1}\right\rangle\left\langle k_{1} k_{2}\right\rangle \ldots\left\langle k_{\ell} p_{1}\right\rangle} .
$$

Substituting (2.19) and (2.20) in (2.18) and matching the result with (2.5) we find

$$
\begin{aligned}
W_{n, m}^{\mathrm{MHV} \times \mathrm{MHV}}(x, k)= & \sum e^{i x_{i_{1}} y_{\ell_{1} \ell_{2}}+i x_{i_{2}} y_{\ell_{2}, \ell_{3}}+\ldots+i x_{i_{s}} y_{\ell_{s}, \ell_{1}}} \\
& \times \frac{\left\langle k_{\ell_{1}-1} k_{\ell_{1}}\right\rangle\left\langle p_{i_{1}} p_{i_{1}-1}\right\rangle\left\langle k_{\ell_{2}-1} k_{\ell_{2}}\right\rangle\left\langle p_{i_{2}} p_{i_{2}-1}\right\rangle \ldots\left\langle k_{\ell_{s}-1} k_{\ell_{s}}\right\rangle\left\langle p_{i_{s}} p_{i_{s}-1}\right\rangle}{\left\langle k_{\ell_{1}} p_{i_{1}}\right\rangle\left\langle p_{i_{1}-1} k_{\ell_{2}-1}\right\rangle\left\langle k_{\ell_{2}} p_{i_{2}}\right\rangle\left\langle p_{i_{2}-1} k_{\ell_{3}-1}\right\rangle \ldots\left\langle k_{\ell_{s}} p_{i_{s}}\right\rangle\left\langle p_{i_{s}-1} k_{\ell_{1}-1}\right\rangle},
\end{aligned}
$$

where the sum covers the same range as in (2.18). Here we used (2.8) to switch to dual momenta in the exponent, e.g. $y_{\ell_{1} \ell_{2}}=k_{\ell_{1}}+\ldots+k_{\ell_{2}-1}$. We recall that for vanishing total momentum $K=\sum_{i=1}^{m} k_{i}=0$, the dual momenta satisfy the periodicity condition $y_{m+1}=y_{1}$. Using this property, we can rewrite the exponential factor in (2.21) in the equivalent form

$$
e^{i y \ell_{s} x_{i_{s}, i_{s-1}}+\ldots+i y \ell_{2} x_{i_{2} i_{1}}+i y \ell_{1} x_{i_{1} i_{s}}} .
$$

We observe that it can be obtained from the original factor by swapping the variables

$$
x_{i_{1}} \leftrightarrow y_{\ell_{s}}, \quad x_{i_{2}} \leftrightarrow y_{\ell_{s-1}}, \quad \ldots, \quad x_{i_{s}} \leftrightarrow y_{\ell_{1}} .
$$

Let us now examine the expression in the second line of (2.21). It depends on two sets of null vectors $p_{i}$ and $k_{i}$ defining the edges of the lightlike Wilson loop and the momenta of the on-shell gluons, respectively. It is straightforward to verify that it is invariant under the swapping of these vectors

$$
k_{\ell_{1}} \leftrightarrow p_{i_{s}}, \quad k_{\ell_{2}} \leftrightarrow p_{i_{s-1}}, \quad \ldots, \quad k_{\ell_{s}} \leftrightarrow p_{i_{1}} .
$$

Putting together (2.23) and (2.24), we immediately conclude that the expression on the right-hand side of (2.21) is invariant under the exchange of the original variables $(x, k)$ with their dual partners $(y, p)$. This yields the duality relation

$$
W_{n, m}^{\mathrm{MHV} \times \mathrm{MHV}}(x, k)=W_{m, n}^{\mathrm{MHV} \times \mathrm{MHV}}(y, p),
$$

in agreement with [20].

\subsection{Duality beyond MHV}

To test the duality relation (2.12) beyond MHV level, we have to take into account the dependence of the Wilson loop form factor (2.1) on the Grassmann variables $\theta_{i}^{A}$ and $\eta_{j A}$. The dependence on $\eta$ comes from the expansion of the on-shell super-state in (2.1) over the states of particles (gluons, gaugino and scalars) with different helicity.

At the same time, the dependence of (2.1) on $\theta$ comes from the expansion of the supersymmetric $n$-gon Wilson loop

$$
\mathcal{W}_{n}=\frac{1}{N} \operatorname{tr}\left(\mathcal{E}_{1 n} \ldots \mathcal{E}_{32} \mathcal{E}_{21}\right)
$$


in powers of $\theta_{i}$ defining the position of vertices of the lightlike $n$-gon in (chiral) superspace. Here the supersymmetric Wilson line $\mathcal{E}_{i+1, i}$ is evaluated along the straight segment connecting the superspace points $\left(x_{i}, \theta_{i}\right)$ and $\left(x_{i+1}, \theta_{i+1}\right)$

$$
\mathcal{E}_{i+1, i}=P \exp \left[-i \int_{0}^{1} d t\left(\frac{1}{2} x_{i, i+1}^{\dot{\alpha} \alpha} \mathcal{A}_{\alpha \dot{\alpha}}(x(t), \theta(t))+\theta_{i, i+1}^{\alpha A} \mathcal{A}_{\alpha A}(x(t), \theta(t))\right)\right]
$$

where $x(t)=x_{i}-x_{i, i+1} t$ and $\theta(t)=\theta_{i}-\theta_{i, i+1} t$. The super-connections $\mathcal{A}$ are subject to the defining on-shell constraints of $\mathcal{N}=4$ SYM [32]. One way of solving them is to fix the non-supersymmetric Wess-Zumino gauge and express the components of $\mathcal{A}$ in terms of the propagating gluon, gaugino and scalar fields [21, 22]

$$
\begin{aligned}
& \mathcal{A}_{\alpha \dot{\alpha}}=A_{\alpha \dot{\alpha}}+i \theta_{\alpha}^{A} \bar{\psi}_{\dot{\alpha} A}+\frac{i}{2 !} \theta_{\alpha}^{A} \theta^{\beta B} D_{\beta \dot{\alpha}} \bar{\phi}_{A B}-\frac{1}{3 !} \epsilon_{A B C D} \theta_{\alpha}^{A} \theta^{\beta B} \theta^{\gamma C} D_{\beta \dot{\alpha}} \psi_{\gamma}^{D}+\ldots \\
& \mathcal{A}_{\alpha A}=\frac{i}{2} \bar{\phi}_{A B} \theta_{\alpha}^{B}-\frac{1}{3 ! !} \epsilon_{A B C D} \theta_{\alpha}^{B} \theta^{\gamma C} \psi_{\gamma}^{D}+\frac{i}{4 ! !} \epsilon_{A B C D} \theta_{\alpha}^{B} \theta^{\beta C} \theta^{\gamma D} F_{\beta \gamma}+\ldots
\end{aligned}
$$

where the dots denote higher-order terms in $\theta$.

Before continuing let us examine the superspace structure we should expect this object to have arising from supersymmetry. The chiral supersymmetry of (2.6) yields the Ward identity

$$
\left(\sum_{i=1}^{n} \frac{\partial}{\partial \theta_{i}^{A}}+\sum_{j=1}^{m}\left|k_{j}\right\rangle \eta_{j, A}\right) W_{n, m}(\{x, \theta\} ;\{k, \eta\})=0 .
$$

The duality relation is expected to hold if the total particle supercharge vanishes, $Q_{A}=$ $\sum_{j=1}^{m}\left|k_{j}\right\rangle \eta_{j A}=0$. Then (2.29) implies that $W_{n, m}$ can be an arbitrary function of $\theta_{i j}^{A}=$ $\theta_{i}^{A}-\theta_{j}^{A}$ and $\eta_{k A}$. In virtue of the $\mathrm{R}$ symmetry, these variables must form SU(4) invariants. The latter are of three different kinds: $\epsilon_{A B C D} \theta_{i i^{\prime}}^{A} \theta_{j j^{\prime}}^{B} \theta_{k k^{\prime}}^{C} \theta_{l l^{\prime}}^{D}, \eta_{i j k l}^{4}=\epsilon^{A B C D} \eta_{i A} \eta_{j B} \eta_{k C} \eta_{l D}$ and $\left(\theta_{i j} \eta_{k}\right)=\theta_{i j}^{A} \eta_{k A}$. The dependence on these invariants simplifies further in the Born approximation.

To compute $W_{n, m}$ in the Born approximation, we substitute (2.26)-(2.28) into the definition (2.1) and retain the contribution at the lowest order in the coupling. Since the dependence on $\theta$ 's comes from the expansion of the bosonic and fermionic connections in (2.28), the number of contributing diagrams and their complexity increases significantly as compared with the MHV case described in the previous subsection. Moreover, the use of the Wess-Zumino gauge (2.28) breaks manifest supersymmetry. This makes the conventional approach impractical.

In this paper we prefer the off-shell formulation of the chiral $\mathcal{N}=4 \mathrm{SYM}$ theory in terms of unconstrained prepotentials in LHC superspace [23], better suited for supersymmetric quantisation. In section 3 we formulate the lightlike Wilson loop in LHC superspace and apply the Feynman rules of [30] to the computation of its form factors.

In this new formulation, $W_{n, m}\left(\left\{x_{i}, \theta_{i}\right\} ;\left\{k_{j}, \eta_{j}\right\}\right)$ is given by a sum of contributions having a similar structure to (2.18), with the important difference that the wedge form factors are replaced by their supersymmetric generalisations depending on the Grassmann variables $\theta_{i}^{A}$ and $\eta_{j A}$. This leads to the following general expression for $W_{n, m}$,

$$
W_{n, m}=\sum e^{i x_{i_{1}} y_{\ell_{1} \ell_{2}}+i x_{i_{2}} y_{\ell_{2}, \ell_{3}}+\ldots+i x_{i_{s}} y_{\ell_{s}, \ell_{1}}} \times e^{\left\langle\theta_{i_{1}} \psi_{\ell_{1} \ell_{2}}\right\rangle+\left\langle\theta_{i_{2}} \psi_{\ell_{2} \ell_{3}}\right\rangle+\ldots+\left\langle\theta_{i_{s}} \psi_{\ell_{s} \ell_{1}}\right\rangle} \times \widehat{W}_{n, m}
$$


which should be compared with (2.21). Here we used shorthand notation for $\left\langle\theta_{i_{1}} \psi_{\ell_{1} \ell_{2}}\right\rangle=$ $\theta_{i_{1}}^{\alpha A}\left(\psi_{\ell_{1}, \alpha A}-\psi_{\ell_{2}, \alpha A}\right)$ with the dual $\psi$-variables defined in (2.8). The sum in (2.30) has the same form as in (2.18) and runs over all possible partitions of $m$ super particles over $s$ clusters. Notice that the function $\widehat{W}_{n, m}$ depends on the choice of partition. The second exponent on the right-hand side of (2.30) is the supersymmetric completion of the first exponent depending on the bosonic variables.

Most importantly, as we show below by exploring the structure of the Feynman diagrams, the function $\widehat{W}_{n, m}$ does not depend on the mixed products of Grassmann variables $\left(\theta_{i j} \eta_{k}\right)$ in the Born approximation. ${ }^{2}$ This allows us to expand $\widehat{W}_{n, m}$ in powers of the two remaining invariants leading to the following relation

$$
\widehat{W}_{n, m}=W_{n, m}^{(0,0)}+\left(W_{n, m}^{(1,0)}+W_{n, m}^{(0,1)}\right)+\left(W_{n, m}^{(2,0)}+W_{n, m}^{(1,1)}+W_{n, m}^{(0,2)}\right)+\ldots
$$

where $W_{n, m}^{(\kappa, \sigma)}$ is a homogenous polynomial in $\theta$ 's and $\eta$ 's of degree $4 \kappa$ and $4 \sigma$, respectively. Schematically, $W_{n, m}^{(\kappa, \sigma)} \sim \theta^{4 \kappa} \eta^{4 \sigma}$. By analogy with the superamplitude, we refer to the terms on the right-hand side of (2.31) with $\kappa+\sigma=k$ as $\mathrm{N}^{k} \mathrm{MHV}$-like. The lowest term of the expansion, $W_{n, m}^{(0,0)}$, defines the MHV-like contribution $W_{n, m}^{\mathrm{MHV} \times \mathrm{MHV}}$ discussed in the previous subsection. Its explicit expression can be read from (2.21).

Substituting (2.30) and (2.31) into (2.12), we can formulate the duality relation in each sector,

$$
W_{n, m}^{(\kappa, \sigma)}(\{x, \theta\} ;\{k, \eta\})=W_{m, n}^{(\sigma, \kappa)}(\{y, \psi\} ;\{p, \omega\}) .
$$

The explicit expressions for $W_{n, m}^{(\kappa, \sigma)}$ for generic $\kappa$ and $\sigma$ are rather complicated even in the Born approximation. Nevertheless, as we show below, the duality relation (2.32) can be verified by matching into each other the diagrams contributing to both sides of (2.32).

\section{Lightlike Wilson loop in LHC superspace}

As mentioned in the introduction, the conventional formulation (2.27) of the chiral supersymmetric Wilson loops, making use of constrained super-connections, is not convenient for quantum calculations. The LHC superspace approach, where the dynamical gauge prepotentials are unconstrained, is much more efficient. In this section we start by a brief summary of the LHC superspace description of $\mathcal{N}=4 \mathrm{SYM}$. Then we present the explicit form of the Wilson loop in LHC superspace, in terms of the two unconstrained gauge prepotentials (the detailed derivation is shown in appendix A). Our formulation is similar to the twistor one of Mason and Skinner in [5] but differs from it in an essential point.

\section{$3.1 \mathcal{N}=4$ super-Yang-Mills in LHC superspace}

Here we recall some basic facts about $\mathcal{N}=4 \mathrm{SYM}$ in LHC superspace (for details see [23]). The theory is formulated in terms of two dynamical chiral superfields (prepotentials),

$$
A^{++}\left(x, \theta^{+}, u\right), \quad A_{\dot{\alpha}}^{+}\left(x, \theta^{+}, u\right) .
$$

\footnotetext{
${ }^{2}$ This does not follow from chiral supersymmetry (2.29) and it would be interesting to understand the symmetry leading to such a structure.
} 
Here $\theta^{+A}=\theta_{\alpha}^{A} u^{+\alpha}$ is a projection of the chiral Grassmann variable with a harmonic variable $u^{+\alpha}$. This commuting spinor variable together with its conjugate $u^{-\alpha}$ form a matrix of the chiral half $\mathrm{SU}(2)_{L}$ of the Euclidean Lorentz group $\mathrm{SO}(4) \sim \mathrm{SU}(2)_{L} \times \mathrm{SU}(2)_{R}$. The harmonic variables $u^{ \pm}$parametrise the coset space $S^{2} \sim \mathrm{SU}(2)_{L} / \mathrm{U}(1)$. The superfields (3.1) are interpreted as infinite harmonic expansions on the sphere, i.e. homogeneous series in the harmonic variables $u^{ \pm}$with fixed U(1) charge. For example, in the expansion of $A_{\dot{\alpha}}^{+}\left(x, \theta^{+}, u\right)=$ $A_{\alpha \dot{\alpha}}(x) u^{+\alpha}+A_{\alpha \beta \gamma \dot{\alpha}}(x) u^{+\alpha} u^{+\beta} u^{-\gamma}+\ldots+O(\theta)$ we find the ordinary gauge field $A_{\alpha \dot{\alpha}}(x)$ and an infinite set of auxiliary higher-spin fields $A_{\alpha \beta \gamma \dot{\alpha}}(x), \ldots$. Note the absence of the other projection $\theta^{-A}=\theta_{\alpha}^{A} u^{-\alpha}$ in (3.1). Such superfields are called chiral-analytic.

The prepotentials have the meaning of the connections for two of the gauge covariant derivatives in the theory, namely

$$
\nabla^{++}=\partial^{++}+A^{++}, \quad \nabla_{\dot{\alpha}}^{+}=\partial_{\dot{\alpha}}^{+}+A_{\dot{\alpha}}^{+}
$$

Here $\partial_{\dot{\alpha}}^{+}=u^{+\alpha} \partial_{\alpha \dot{\alpha}}$ is a projection of the space-time derivative $\partial_{x}$ while $\partial^{++}=u^{+\alpha} \partial / \partial u^{-\alpha}$ is one of the two covariant derivatives on $S^{2}$. These derivatives transform with a gauge parameter of the chiral-analytic type,

$$
\nabla \rightarrow e^{\Lambda\left(x, \theta^{+}, u\right)} \nabla e^{-\Lambda\left(x, \theta^{+}, u\right)}
$$

The remaining gauge connections can be constructed from the prepotentials by solving the various super-curvature constraints. In particular, the projected spinor derivative $\partial_{A}^{+}=$ $u^{+\alpha} \partial / \partial \theta^{\alpha A}$ commutes with the gauge parameter $\Lambda\left(x, \theta^{+}, u\right)$, hence it needs no connection, $\nabla_{A}^{+}=\partial_{A}^{+}$.

The action of the theory consists of two terms,

$$
S_{\mathcal{N}=4 S Y M}=\int d u d^{4} x d^{4} \theta^{+} L_{C S}\left(x, \theta^{+}, u\right)+\int d^{4} x d^{8} \theta L_{Z}(x, \theta) .
$$

The first term in (3.4) is of the Chern-Simons type,

$$
L_{\mathrm{CS}}\left(x, \theta^{+}, u\right)=\operatorname{tr}\left(A^{++} \partial^{+\dot{\alpha}} A_{\dot{\alpha}}^{+}-\frac{1}{2} A^{+\dot{\alpha}} \partial^{++} A_{\dot{\alpha}}^{+}+A^{++} A^{+\dot{\alpha}} A_{\dot{\alpha}}^{+}\right)
$$

and it describes the self-dual sector of the theory [33]. The second term in (3.4) involves only the prepotential $A^{++}$in a non-polynomial way [24,34],

$$
L_{\mathrm{Z}}=\operatorname{tr} \sum_{n=2}^{\infty} \frac{(-1)^{n}}{n} \int d u_{1} \ldots d u_{n} \frac{A^{++}\left(x, \theta_{1}^{+}, u_{1}\right) \ldots A^{++}\left(x, \theta_{n}^{+}, u_{n}\right)}{\left\langle u_{1}^{+} u_{2}^{+}\right\rangle \ldots\left\langle u_{n}^{+} u_{1}^{+}\right\rangle}
$$

where $\theta_{i}^{+A}=\theta^{\alpha A}\left(u_{i}\right)_{\alpha}^{+}$with $i=1, \ldots, n$ and $\left\langle u_{i}^{+} u_{j}^{+}\right\rangle=u_{i}^{+\alpha} \epsilon_{\alpha \beta} u_{j}^{+\beta}$. This Lagrangian is local in $(x, \theta)$ space but non-local in the harmonic space (each copy of $A^{++}$depends on its own harmonic variable). The gauge coupling constant $g$ can be restored by redefining $A \rightarrow g A$ and $L \rightarrow g^{-2} L$.

In this paper we are dealing with form factors, so we need to define the supersymmetric on-shell states. A detailed discussion can be found in [30], here we only recall that 
the super-wave functions of the prepotentials $A$ in the state with (super)momentum $(k, \eta)$ have the form

$$
\left\langle k, \eta\left|A^{++}\left(x, \theta^{+}, u\right)\right| 0\right\rangle=\delta^{2}(k, u) e^{i k x+\langle k \theta\rangle \eta}, \quad\left\langle k, \eta\left|A_{\dot{\alpha}}^{+}\left(x, \theta^{+}, u\right)\right| 0\right\rangle=0
$$

provided we quantise the theory in the light-cone gauge (B.1). The harmonic delta function $\delta^{2}(k, u)$ identifies the harmonic variable of the field with the chiral spinor momentum, $u_{\alpha}^{+}=k_{\alpha}$. Notice that only the prepotential $A^{++}$has a non-trivial wave function, while $A_{\dot{\alpha}}^{+}$ does not appear in external states.

\subsection{Chiral Wilson loop in LHC superspace}

Now, the question arises how to reformulate the Wilson loop (2.26), (2.27) in terms of the prepotentials? The detailed answer is given in appendix A, here we just summarise it.

The chiral lightlike Wilson loop in LHC superspace takes the following form:

$$
\mathcal{W}_{n}=\frac{1}{N} \operatorname{tr} \prod_{i=1}^{n} U\left(x_{i}, \theta_{i} ; p_{i}, p_{i-1}\right) E_{i+1, i}
$$

Here the so-called bilocal bridge

$$
U\left(x, \theta ; p_{2}, p_{1}\right)=1+\sum_{n=1}^{\infty}(-1)^{n} \int d u_{1} \ldots d u_{n} \frac{\left\langle p_{2} p_{1}\right\rangle A^{++}(1) \ldots A^{++}(n)}{\left\langle p_{2} u_{1}^{+}\right\rangle\left\langle u_{1}^{+} u_{2}^{+}\right\rangle \ldots\left\langle u_{n}^{+} p_{1}\right\rangle}
$$

resembles the interaction Lagrangian (3.6). The bridges glue together adjacent Wilson line segments in (3.8),

$$
E_{i+1, i}=P \exp \left\{-\frac{i}{2} \int_{0}^{1} d t \tilde{p}_{i}^{\dot{\alpha}} A_{\dot{\alpha}}^{+}\left(x_{i}-t \tilde{p}_{i} p_{i},\left\langle p_{i} \theta_{i}\right\rangle,\left|p_{i}\right\rangle\right)\right\} .
$$

We remark that in the expression for the Wilson loop (3.8) the prepotential $A^{++}$appears only at the cusps of the Wilson loop contour via the bilocal bridge $U(3.9)$, while the other prepotential $A_{\dot{\alpha}}^{+}$contributes only through the edges of the contour.

We would like to emphasise that the definition of the Wilson loop (3.8) differs from the twistor formulation of Mason and Skinner [5]. They have the analog of the bilocal bridge $U$ (called 'parallel propagator') but not the Wilson line segments $E_{i+1, i}$ (see the discussion in appendix A.3). We believe that their definition is not gauge invariant and hence it is incomplete. Still, the result of their calculation of the NMHV Wilson loop is correct, for a reason which will become clear at the end of section 5. However, as we show in this paper, the Wilson line segments in (3.8) are indispensable for obtaining a gauge-invariant result for the Wilson loop form factor.

\section{Diagrammatic approach to the duality}

In this section we illustrate the duality (2.32) in the simplest MHV $\times$ MHV case. It corresponds to the first term on the right-hand side of (2.31) which has the lowest Grassmann degree $(\kappa=0, \sigma=0)$. We apply the Feynman rules from appendix B to the calculation of 

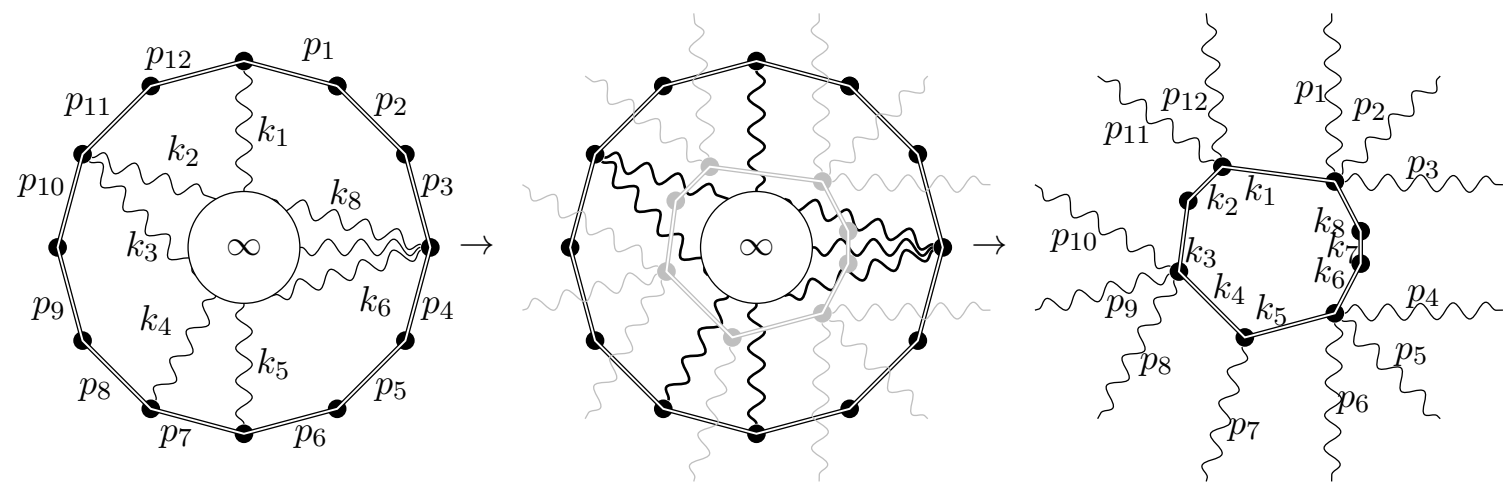

Figure 3. The left figure represents a planar Born-level diagram for the Wilson loop form factor $W_{12,8}^{(0,0)}$. The external particles are coming from infinity which is chosen inside the Wilson loop contour. The right figure represents a diagram for $W_{8,12}^{(0,0)}$ where the variables specifying the Wilson loop contour and the external particles are swapped. Here infinity is chosen to lie outside the Wilson loop contour. In the middle figure the two diagrams are superimposed so that the planar graph duality is manifest.

the Wilson loop form factor defined in (3.8), in the planar limit and to the lowest order in the coupling and rederive the result (2.21). This example illustrates both the graph duality and the simplicity of the LHC computation by applying the effective rules of appendix B.2.

We end the section by a discussion of the general structure of the non-MHV diagrams.

\subsection{MHV example}

As follows from the definition of the Wilson loop (3.8)-(3.10), to the lowest degree in the Grassmann variables, the Born-level contribution only comes from diagrams without internal propagators and interaction vertices and with the prepotential $A^{++}$replaced by the wave function (B.10). Indeed, the propagators of the prepotentials $A^{++}$and $A_{\dot{\alpha}}^{+}$given by (B.9), (B.12), (B.13) and (B.14) are nilpotent (either $\sim \theta^{4}$ or $\sim \eta^{4}$ ) and increase the Grassmann degree. This leaves us with only one type of diagram illustrated in figure 3.

Here in the diagram on the left-hand side we draw all external legs inside the Wilson loop contour. These legs are ordered according to (2.2) and they end at a point that we call 'infinity'. This graph contains $n=12$ edges and $m=8$ external particles and contributes to $F_{12,8}$. In the second diagram in figure 3 we show the planar dual graph faintly superimposed. For every face of the original graph we draw a vertex, then we join the vertices up by appropriate edges going through the boundaries of two faces as described above. This results in the third diagram which we recognise as a valid $\mathrm{MHV} \times \mathrm{MHV}$ diagram contributing to $F_{8,12}$ with all external legs outside the Wilson loop.

Let us compute the graph expressions using the simple rules from appendix B.2. First consider the left diagram in figure 3. There are five non-trivial cusps of the Wilson loop 
emitting particles. Using (B.7) and (B.11) we obtain the following contribution to $F_{12,8}$

$$
\begin{aligned}
F_{12,8}= & \frac{e^{i k_{1} x_{1}+Q_{1} \theta_{1}}\left\langle p_{12} p_{1}\right\rangle}{\left\langle p_{1} k_{1}\right\rangle\left\langle k_{1} p_{12}\right\rangle} \times \frac{e^{i\left(k_{2}+k_{3}\right) x_{11}+\left(Q_{2}+Q_{3}\right) \theta_{11}}\left\langle p_{10} p_{11}\right\rangle}{\left\langle p_{11} k_{2}\right\rangle\left\langle k_{2} k_{3}\right\rangle\left\langle k_{3} p_{10}\right\rangle} \\
& \times \frac{e^{i k_{4} x_{8}+Q_{4} \theta_{8}}\left\langle p_{7} p_{8}\right\rangle}{\left\langle p_{8} k_{4}\right\rangle\left\langle k_{4} p_{7}\right\rangle} \times \frac{e^{i k_{5} x_{7}+Q_{5} \theta_{7}}\left\langle p_{6} p_{7}\right\rangle}{\left\langle p_{7} k_{5}\right\rangle\left\langle k_{5} p_{6}\right\rangle} \times \frac{e^{i\left(k_{6}+k_{7}+k_{8}\right) x_{4}+\left(Q_{6}+Q_{7}+Q_{8}\right) \theta_{4}}\left\langle p_{3} p_{4}\right\rangle}{\left\langle p_{4} k_{6}\right\rangle\left\langle k_{6} k_{7}\right\rangle\left\langle k_{7} k_{8}\right\rangle\left\langle k_{8} p_{3}\right\rangle} .
\end{aligned}
$$

where $Q_{i} \theta_{j} \equiv \eta_{i A}\left\langle k_{i} \theta_{j}^{A}\right\rangle$. The dependence on the Grassmann variables follows the similar bosonic variables exponents. Substituting $F_{12,8}$ into (2.5) and (2.30) we obtain the corresponding contribution to $\widehat{W}_{12,8}$

$$
\widehat{W}_{12,8}=\frac{\left\langle k_{8} k_{1}\right\rangle\left\langle k_{1} k_{2}\right\rangle\left\langle k_{3} k_{4}\right\rangle\left\langle k_{4} k_{5}\right\rangle\left\langle k_{5} k_{6}\right\rangle \times\left\langle p_{12} p_{1}\right\rangle\left\langle p_{10} p_{11}\right\rangle\left\langle p_{7} p_{8}\right\rangle\left\langle p_{6} p_{7}\right\rangle\left\langle p_{3} p_{4}\right\rangle}{\left\langle p_{1} k_{1}\right\rangle\left\langle k_{1} p_{12}\right\rangle\left\langle p_{11} k_{2}\right\rangle\left\langle k_{3} p_{10}\right\rangle\left\langle p_{8} k_{4}\right\rangle\left\langle k_{4} p_{7}\right\rangle\left\langle p_{7} k_{5}\right\rangle\left\langle k_{5} p_{6}\right\rangle\left\langle p_{4} k_{6}\right\rangle\left\langle k_{8} p_{3}\right\rangle} .
$$

Let us now look at the right diagram in figure 3 . It depends on the variables $\left(y_{j}, \psi_{j}\right)$ defining the Wilson loop contour and the variables $\left(p_{i}, \omega_{i}\right)$ specifying the external particles. Using the effective Feynman rules, we obtain the following contribution to $F_{8,12}$ :

$$
\begin{aligned}
F_{8,12}= & \frac{e^{i\left(p_{1}+p_{2}+p_{3}\right) y_{1}+\left(\tilde{Q}_{1}+\tilde{Q}_{2}+\tilde{Q}_{3}\right) \psi_{1}}\left\langle k_{8} k_{1}\right\rangle}{\left\langle k_{8} p_{3}\right\rangle\left\langle p_{3} p_{2}\right\rangle\left\langle p_{2} p_{1}\right\rangle\left\langle p_{1} k_{1}\right\rangle} \times \frac{e^{i\left(p_{11}+p_{12}\right) y_{2}+\left(\tilde{Q}_{11}+\tilde{Q}_{12}\right) \psi_{2}}\left\langle k_{1} k_{2}\right\rangle}{\left\langle k_{1} p_{12}\right\rangle\left\langle p_{12} p_{11}\right\rangle\left\langle p_{11} k_{2}\right\rangle} \\
& \times \frac{e^{i\left(p_{8}+p_{9}+p_{10}\right) y_{4}+\left(\tilde{Q}_{8}+\tilde{Q}_{9}+\tilde{Q}_{10}\right) \psi_{4}}\left\langle k_{3} k_{4}\right\rangle}{\left\langle k_{3} p_{10}\right\rangle\left\langle p_{10} p_{9}\right\rangle\left\langle p_{9} p_{8}\right\rangle\left\langle p_{8} k_{4}\right\rangle} \times \frac{e^{i p_{7} y_{5}+\tilde{Q}_{7} \psi_{5}}\left\langle k_{4} k_{5}\right\rangle}{\left\langle k_{4} p_{7}\right\rangle\left\langle p_{7} k_{5}\right\rangle} \\
& \times \frac{e^{i\left(p_{4}+p_{5}+p_{6}\right) y_{6}+\left(\tilde{Q}_{4}+\tilde{Q}_{5}+\tilde{Q}_{6}\right) \psi_{6}}\left\langle k_{5} k_{6}\right\rangle}{\left\langle k_{5} p_{6}\right\rangle\left\langle p_{6} p_{5}\right\rangle\left\langle p_{5} p_{4}\right\rangle\left\langle p_{4} k_{6}\right\rangle},
\end{aligned}
$$

where $\tilde{Q}_{i} \psi_{j} \equiv \omega_{i}^{A}\left\langle p_{i} \psi_{j A}\right\rangle$. Substituting this expression into (2.5) and (2.30) we find that its contribution to $\widehat{W}_{8,12}$ is precisely equal to (4.2),

$$
\widehat{W}_{8,12}=\widehat{W}_{12,8} \text {. }
$$

This example illustrates the general diagrammatic proof of the duality in the MHV case: there are mixed $\left\langle k_{i} p_{j}\right\rangle$ brackets, common to both the graph and its dual. Then the missing $\left\langle k_{i} k_{j}\right\rangle$ brackets in the denominator on one side become explicit numerator terms from the Wilson loop vertices on the other, and vice versa for the $\left\langle p_{i} p_{j}\right\rangle$ brackets.

The exponential factors can be seen to agree in general, also diagrammatically. Using $k_{j}=y_{j}-y_{j+1}$ we find that there is an exponent $e^{i x_{i} y_{j}}$ in the left diagram if and only if the face $y_{j}$ has a corner $x_{i}$. In the dual picture faces and vertices are swapped, but the result is unchanged. The Grassmann exponents follow the same pattern.

\subsection{Classification of diagrams}

Going beyond the MHV level, we have to consider diagrams containing propagators and interaction vertices. An example of such a diagram is shown in figure 1. The left diagram contains two propagators connecting cusp points and one propagator connecting a cusp point with a vertex of emission of two particles. The former produce a factor $O\left(\theta^{8}\right)$ and the latter yields a factor $O\left(\eta^{4}\right)$. As a result, this diagram describes an $\mathrm{N}^{2} \mathrm{MHV} \times \mathrm{NMHV}$ 
contribution. Similarly, the contribution of the right diagram in figure 1 is $\mathrm{NMHV} \times \mathrm{N}^{2} \mathrm{MHV}$ like. In general, a diagram with $\kappa+\sigma$ propagators (dash lines) and $\sigma$ emission vertices (white dots) gives rise to an $\mathrm{N}^{\kappa} \mathrm{MHV} \times \mathrm{N}^{\sigma} \mathrm{MHV}$ contribution in the Born approximation.

The duality essentially works diagram by diagram, although there are a few subtleties which will be discussed in the next section. Indeed the duality can be seen very straightforwardly at the diagrammatic level and is essentially a planar graph duality.

Planarity in this context is slightly non-trivial since on the one hand we have external legs coming from infinity and on the other, position space propagators between the cusp points. In the planar limit the only diagrams that survive are those which can be drawn with all external legs and all internal propagators "outside" the Wilson loop contour, without any of the lines crossing, and with the external legs going to infinity (see left diagram in figure 1). There is an alternative description where we move the source of the external legs "infinity" to a point inside the Wilson loop and insist that all legs and internal propagators lie inside the Wilson loop without any lines crossing (see the right diagram in figure 1).

To obtain the dual of a contributing graph, draw a vertex inside each face, and connect vertices by lines going through the boundaries of the faces: if the boundary of two faces is a leg (wavy line), connect the corresponding vertices with a Wilson line (double line), if the boundary is a Wilson line connect with a leg and if the boundary is a propagator (dash line), connect them with propagators (see the middle diagram in figures 1 and 3).

The resulting dual graph will be a valid graph contributing to the dual Wilson loop form factor (but in the opposite description i.e. if the original graph had all propagators outside the Wilson loop contour, the dual graph has all propagators inside). Furthermore, the expressions for the two graphs are identical after swapping the variables appropriately.

Let us note that the case $W_{n, 0}^{(\kappa, 0)}=W_{0, n}^{(0, \kappa)}$ corresponds to the duality between the vacuum expectation value of the $n$-gon Wilson loop and the $\mathrm{N}^{\kappa} \mathrm{MHV} n$-particle amplitude. The diagrammatic interpretation of this duality in terms of momentum twistor variables has been discussed in [5].

\section{Duality between $\mathrm{NMHV} \times \mathrm{MHV}$ and $\mathrm{MHV} \times \mathrm{NMHV}$}

We now move on to consider diagrams from the second term in eq. (2.31), i.e. $\kappa+\sigma=1$, which come in two types $(\kappa, \sigma)=(0,1)$ or $(1,0)$. Each type consists of diagrams with a single propagator. The diagrams of the first type $(0,1)$ have Grassmann structure $O\left(\eta^{4}\right)$ and contain an emission vertex (white blob). According to the Feynman rules (see (B.12) and (B.13)) this vertex is connected by a propagator (dash line) either to a cusp point (black blob) or to an edge of the Wilson loop contour (double line). The diagrams of the second type $(1,0)$ have Grassmann structure $O\left(\theta^{4}\right)$. They contain one propagator which is stretched either between two cusps or between a cusp and an edge of the Wilson loop contour. We call diagrams where all propagators end on cusps/vertices "cusp diagrams". Diagrams involving propagators ending on edges are called "edge diagrams". ${ }^{3}$

\footnotetext{
${ }^{3}$ Note that only one end of a propagator can be on a Wilson loop edge, since there is no propagator between two prepotentials $A_{\dot{\alpha}}^{+}$, see appendix (B).
} 
In this section we carefully examine the NMHV-like case by focussing on the sector of a general Feynman diagram involving the propagator. We first examine the cusp diagrams before turning to the edge diagrams, show how spurious poles cancel and in the process allow the duality to hold in a surprising and non-trivial fashion.

\subsection{Cusp diagrams}

First we consider the diagrams contributing to the form factor $W_{n, m}^{(1,0)}$, eq. (2.31) in the Born approximation. These diagrams contain $n$ cusps (black blobs), $m$ external states (wave lines) and one propagator (dash line). Focussing on the part of the diagram containing the propagator we have ${ }^{4}$

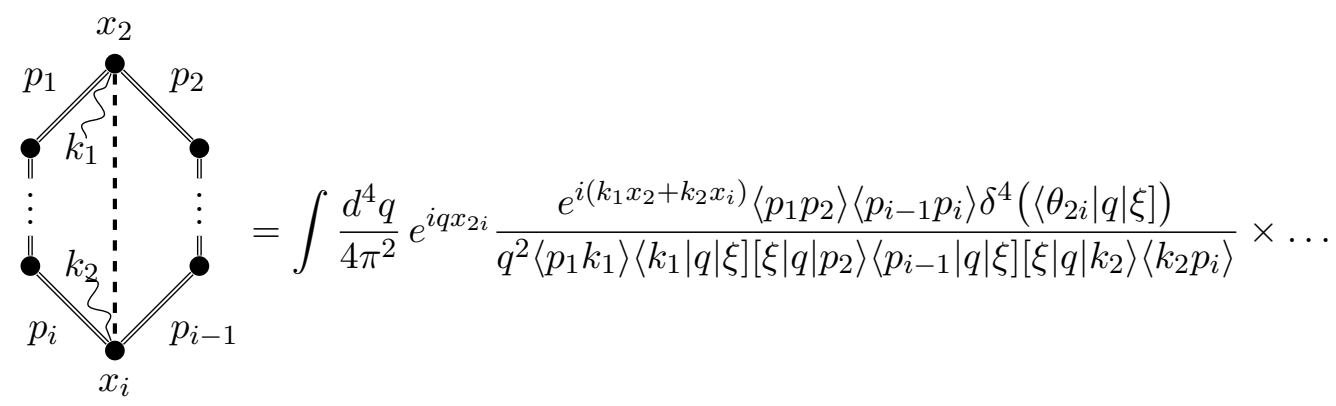

where $q$ is the momentum that flows through the dash line. Here and in all expressions below we drop the exponential dependence on the Grassmann variables $e^{\eta_{1}\left\langle k_{1} \theta_{2}\right\rangle+\eta_{2}\left\langle k_{2} \theta_{i}\right\rangle}$ which follows the similar exponential factor of the bosonic variables. The dots on the right-hand side of (5.1) denote the contribution of the remaining cusps which are the same as in the MHV case.

Now consider cusp diagrams contributing to $W_{m, n}^{(0,1)}$. Again focussing on the piece containing the propagator, the dual to the above diagram is:

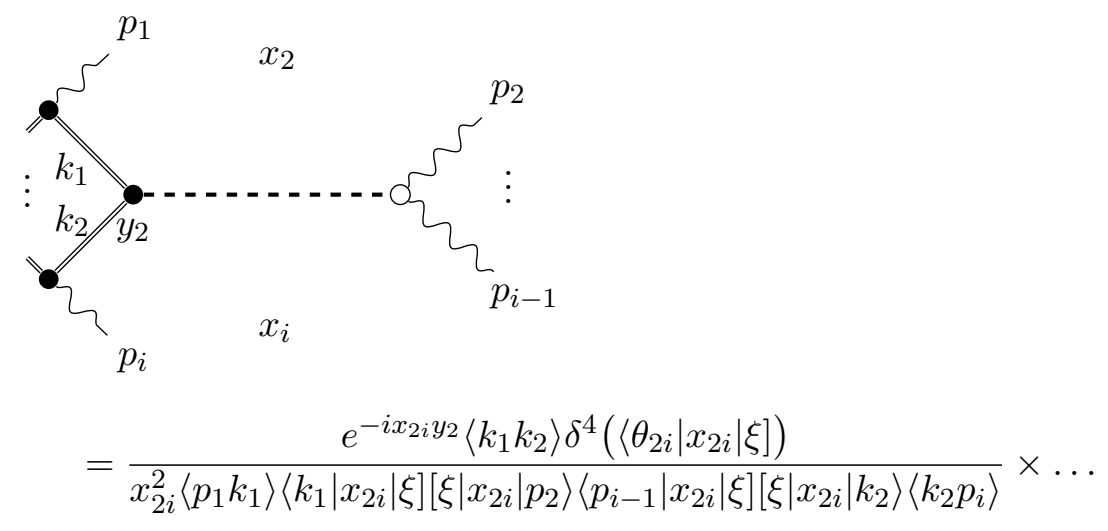

\footnotetext{
${ }^{4}$ We use this diagram to illustrate the general case. The most generic diagram would have an arbitrary number of legs (or none) in place of $k_{1}$ and $k_{2}$.
} 
Here we have replaced the momentum through the propagator $p_{2}+\cdots+p_{i-1}$ by the dual variable $x_{2 i}$ and similarly the supermomentum by $\theta_{2 i}$.

The expressions (5.1) and (5.2) depend on the gauge fixing spinor $\xi$ (see eq. (B.1)) which generate spurious complex poles, e.g. $\left\langle k_{1}|q| \xi\right]=0$ in (5.2). Below we demonstrate that the $\xi$-dependence as well as the spurious poles cancel in the sum of all diagrams.

Notice that the two expressions (5.1) and (5.2) look very similar. In fact, if we replaced $q$ in (5.1) by $x_{2 i}$, then the integrand of the Fourier integral would be identical to the expression in (5.2) (up to $\left\langle k_{r} k_{r+1}\right\rangle$ and $\left\langle p_{r} p_{r+1}\right\rangle$ factors which we expect from the duality relations (2.5) and (2.12)). ${ }^{5}$ Indeed if we were allowed to perform the Fourier transform in (5.1) in Euclidean space, then we would simply replace $q$ by $x_{2 i}$ everywhere according to eq. (C.4). Thus if we were in Euclidean space, (5.1) and (5.2) would give identical expressions (up to the Parke-Taylor factors) leading to the required duality between diagrams. At the moment however we cannot yet justify Wick rotation to Euclidean space as (5.1) has spurious complex poles in $q$-space preventing this. It will become possible after we take into account the edge diagrams.

\subsection{Edge diagrams}

Besides the cusp diagrams we also have edge diagrams with propagators ending on (and being integrated along) edges of the Wilson loop contour. These appear in both $W_{n, m}^{(1,0)}$ and $W_{m, n}^{(0,1)}$.

An example of such a contribution to $W_{n, m}^{(1,0)}$ is

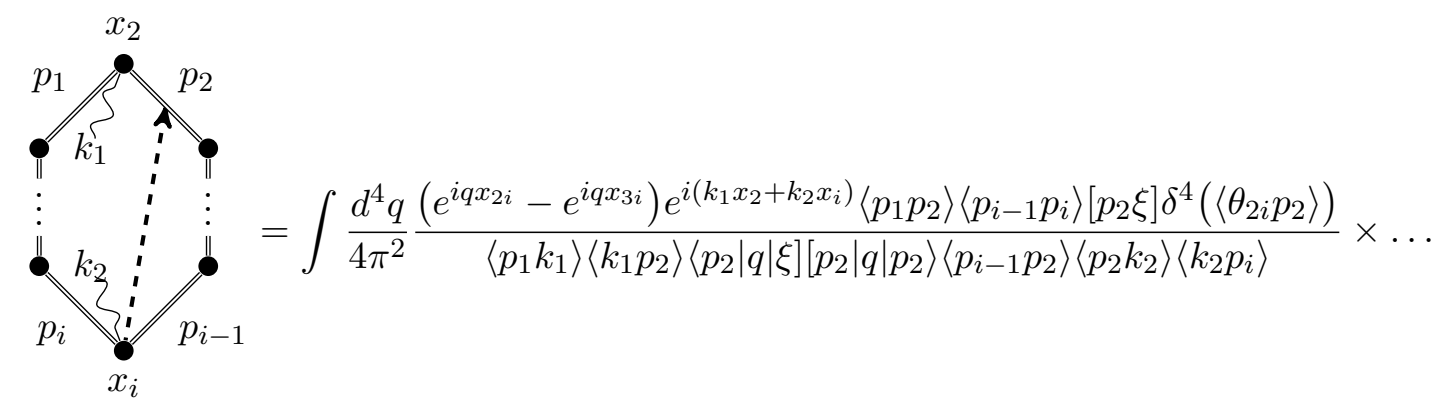

We note here that, again if we could perform the Fourier integration in Euclidean space, we would get two terms coming from the two exponents in the first factor. The first term is obtained by replacing $q$ in the integrand by $x_{2 i}$ and the second one replacing $q$ by $x_{3 i}$, see eq. (C.6). But these two terms are equal (and opposite) since $x_{3 i}=x_{2 i}+x_{32}=x_{2 i}-p_{2}$ and $q$ appears everywhere contracted with a $p_{2}$. Thus as a Euclidean Fourier integral we get from (5.3) a vanishing result and indeed we will find no corresponding dual diagram as we will discuss shortly.

However, as a Fourier integral in Minkowski space, (5.3) is non-vanishing and plays an important role in the cancellation of spurious poles which ultimately allows for the Wick rotation to Euclidean space. We will take a closer look at spurious pole cancellation in the

\footnotetext{
${ }^{5}$ We have displayed only part of the exponential factors in (5.1) and (5.2), the rest goes into the ellipsis. To compare them, in (5.1) we rewrite $k_{1} x_{2}+k_{2} x_{i}=-y_{2} x_{2 i}+\ldots$, as in (5.2).
} 
next subsection, but for the moment we leave all the diagrams contributing to $W_{n, m}^{(1,0)}$ in the form of Fourier integrals.

There are also edge diagrams contributing to $W_{m, n}^{(0,1)}$ for example

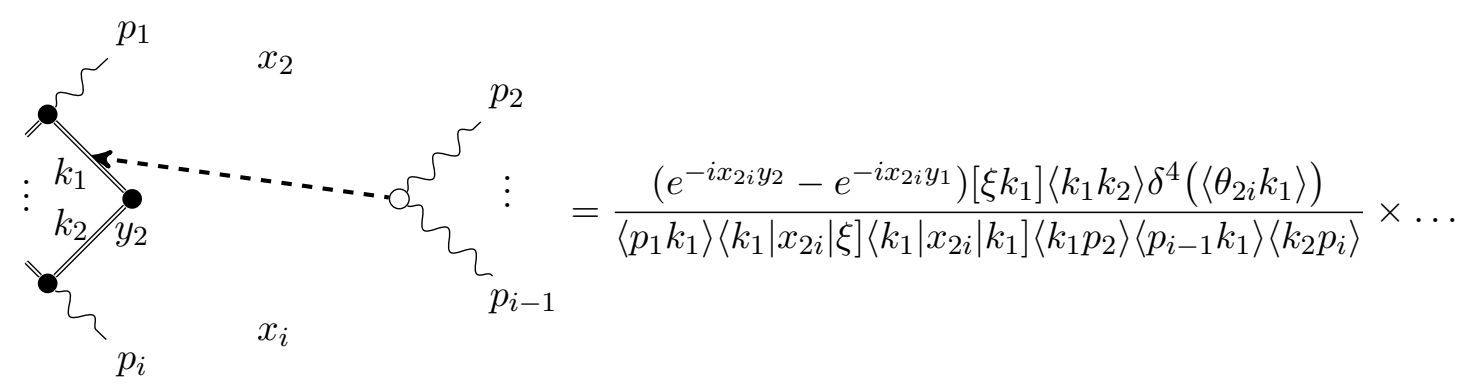

This type of diagram also plays a crucial role in the cancellation of spurious poles. Again there is no corresponding dual diagram. Intriguingly however, if we used the duality to translate this diagram into an expression contributing to the dual diagram $W_{n, m}^{(1,0)}$ we would obtain an integral which evaluates to zero in Minkowski space. However this vanishing Minkowski integral plays a crucial role in the cancellation of leftover spurious poles of $W_{n, m}^{(1,0)}$ at the level of the Fourier integrand. Armed with these additional terms we then have a Fourier integrand with no remaining spurious poles, and we can hence Wick rotate and do the Fourier integration. After this has been performed these "fake" terms contribute a non-vanishing result! We will see all this more explicitly in the next two subsections.

We end this subsection by a comment on the vacuum expectation value of the lightlike Wilson loop $\left\langle 0\left|\mathcal{W}_{n}\right| 0\right\rangle$ at NMHV level. It corresponds to diagrams of the type (5.1) and (5.3) without external legs. In [5] the same object has been calculated in the twistor framework using the incomplete expression (A.20) for the Wilson loop. Evidently, this can account only for the cusp diagrams but the edge diagrams are missing. Nevertheless, the final result is correct. The reason for this is that the authors of [5] work in Euclidean space where the missing edge diagrams vanish, as we have shown. However, the edge diagrams become very important when we consider the form factors of the Wilson loop (2.1).

\subsection{Spurious pole cancellation}

In this subsection we take the generic diagrams (5.1) and (5.2) contributing to $W_{n, m}^{(1,0)}$ and $W_{m, n}^{(0,1)}$, respectively, examine their spurious poles and show how they cancel. We begin with $W_{m, n}^{(0,1)}$ since there things are more straightforward.

$\mathbf{M H V} \times \mathbf{N M H V}$ sector. Diagram (5.2) contains four spurious poles. We consider each pole in turn.

It is convenient to associate the spurious poles of (5.2) with the four angles formed by the propagator and the two pairs of lines $\left(k_{1}, k_{2}\right)$ and $\left(p_{2}, p_{i-1}\right)$ attached to each end. First we consider the pole at $\left[\xi\left|x_{2 i}\right| p_{2}\right\rangle=0$ (associated with the upper right angle). By setting $\mid \xi]=x_{2 i}\left|p_{2}\right\rangle$ it is straightforward to find the residue of (5.2) at this pole. We can then check that the residue is cancelled by the pole of a nearby diagram with the leg $p_{2}$ 
attached to the other end of the propagator. Diagrammatically, displaying the residue by filling in the associated angle we have

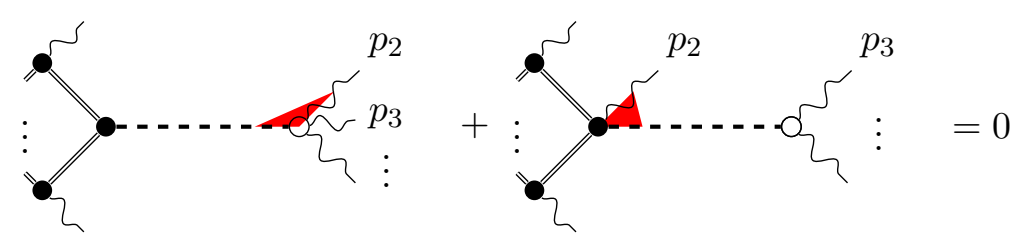

The pole at $\left\langle p_{i-1}\left|x_{2 i}\right| \xi\right]=0$ is cancelled by a very similar mechanism, i.e. the nearby diagram with leg $p_{i-1}$ attached to the other end of the propagator cancels this pole.

Now we consider the pole at $\left\langle k_{1}\left|x_{2 i}\right| \xi\right]=0$ (associated with the upper left angle). By setting $\mid \xi]=x_{2 i}\left|k_{1}\right\rangle$ we can obtain its residue and it is straightforward to check that this cancels the corresponding residue from the first term of the nearby edge diagram (5.4). The second term of the edge diagram then cancels a spurious pole from another nearby cusp diagram. Diagrammatically:

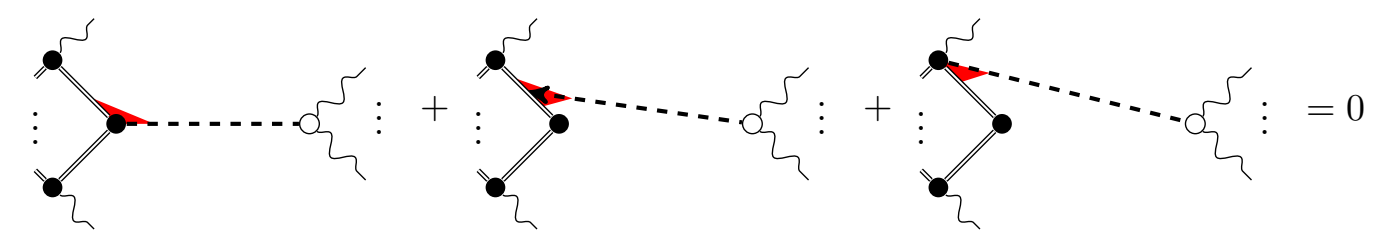

The spurious pole of (5.2) at $\left[\xi\left|x_{2 i}\right| k_{2}\right\rangle=0$ is cancelled by a similar mechanism (essentially the above picture reflected in the horizontal axis.)

We have thus cancelled all spurious poles of the diagram (5.2) using nearby diagrams. Of course each new diagram (apart from the edge diagram) will introduce new spurious poles, which are then cancelled by further nearby diagrams etc. In this way we see that all spurious poles are cancelled up to certain special boundary cases which we consider in appendix D.

$\mathbf{N M H V} \times \mathbf{M H V}$ sector. We now consider the spurious poles of $W_{n, m}^{(1,0)}$ taking the diagram in (5.1) as a suitably generic example. Again there are four spurious poles which we consider in turn.

First consider the pole at $\left[\xi|q| p_{2}\right\rangle=0$. This is shared by the nearby edge diagram (5.3). By considering the limit $\left[\xi \mid \rightarrow\left\langle p_{2}\right| q\right.$ we can find the residue at this pole of both (5.1) and (5.3). We find that the pole of the first term of (5.3) exactly cancels the pole of (5.1). The second term of (5.3) on the other hand cancels a pole of a nearby cusp diagram. Diagrammatically, denoting the pole of a particular diagram by filling in the angle between 
the relevant propagator and neighbouring edge we find

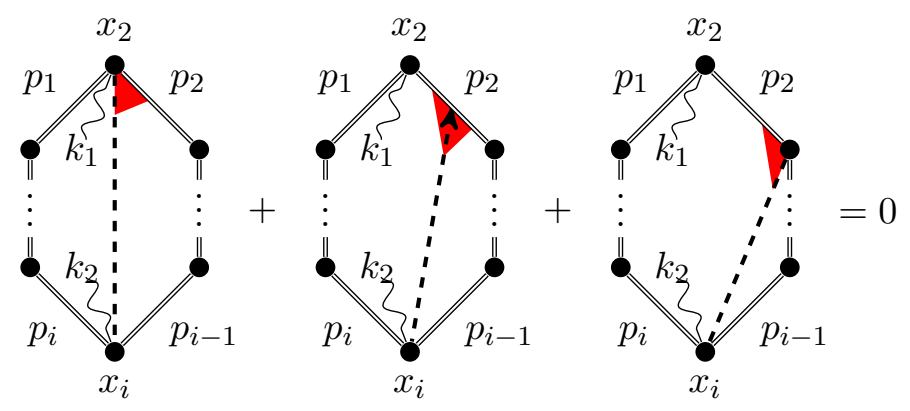

The spurious pole of (5.1) at $\left[\xi|q| p_{i-1}\right\rangle=0$ is cancelled similarly (by essentially a reflection of (5.7) about the horizontal axis).

However now consider the spurious pole at $\left\langle k_{1}|q| \xi\right]=0$. There is no nearby diagram which can cancel this spurious pole at the level of the integrand. One is tempted to consider the diagram with leg $k_{1}$ shifted down so that it is attached to vertex $x_{i}$, but the two diagrams have different exponential factors, $e^{i k_{1} x_{2}}$ and $e^{i k_{1} x_{i}}$, preventing the cancellation. At first sight, this leads to an apparent breakdown of gauge invariance in this sector!

The solution to this puzzle in fact comes from examining the corresponding cancellation occurring in the dual picture described above. There we had a corresponding pole at $\left\langle k_{1}\left|x_{2 i}\right| \xi\right]=0$ which was cancelled by the edge diagram (5.4). Translating this via the duality ${ }^{6}$ suggests we consider the expression

$$
I:=\int \frac{d^{4} q}{4 \pi^{2}} e^{i q x_{2 i}} \frac{\left(e^{i\left(k_{1} x_{2}+k_{2} x_{i}\right)}-e^{i\left(k_{1}+k_{2}\right) x_{i}}\right)\left[\xi k_{1}\right]\left\langle p_{1} p_{2}\right\rangle \delta^{4}\left(\left\langle\theta_{2 i} k_{1}\right\rangle\right)}{\left\langle p_{1} k_{1}\right\rangle\left\langle k_{1}|q| \xi\right]\left\langle k_{1}|q| k_{1}\right]\left\langle k_{1} k_{2}\right\rangle\left\langle k_{1} p_{2}\right\rangle\left\langle p_{i-1} k_{1}\right\rangle\left\langle k_{2} p_{i}\right\rangle} \times \cdots=0 .
$$

Firstly we note that as an integral in Minkowski space $I$ vanishes and thus we are perfectly at liberty to add the integral (5.8) to $W_{n, m}^{(1,0)}$. The identity $I=0$ can be seen straightforwardly by observing that the contributions of the two terms inside the parentheses cancel against each other after shifting $q \rightarrow q+k_{1}$ in the second term (since $\left\langle k_{1}\right| q=\left\langle k_{1}\right|\left(q+k_{1}\right)$ ).

Secondly we note that the integrand of (5.8) is precisely what is needed to recover gauge invariance of the integrand of $W_{n, m}^{(1,0)}$ : the spurious pole of the first term at $\left\langle k_{1}|q| \xi\right]=0$ cancels the spurious pole of the diagram (5.1). Furthermore the other term similarly cancels the pole of the diagram with $k_{1}, k_{2}$ both coming from point $x_{i}$. Thus, for the relevant poles

\footnotetext{
${ }^{6}$ More precisely, to obtain this dual expression as a Fourier integral, we take the expression (5.4), replace $x_{2 i}$ with $q$ in the rational terms, leaving the exponential terms as they are, multiply by $e^{i q x_{2 i}}$ and integrate over $d^{4} q$. The careful reader will note that the exponents of (5.4) and (5.8) are different. In fact the two displayed exponentials are related via $\left(e^{-i x_{2 i} y_{2}}-e^{-i x_{2 i} y_{1}}\right)=e^{-i y_{1} x_{1}}\left(e^{i\left(k_{1} x_{2}+k_{2} x_{i}\right)}-e^{i\left(k_{1}+k_{2}\right) x_{i}}\right) e^{i y_{3} x_{i}}$ and we have simply absorbed the factors $e^{-i y_{1} x_{1}} e^{i y_{3} x_{i}}$ into the ellipsis. If the full exponential factors for the entire expressions were written out in both cases they would precisely agree (see footnote 5 ).
} 
we have diagrammatically

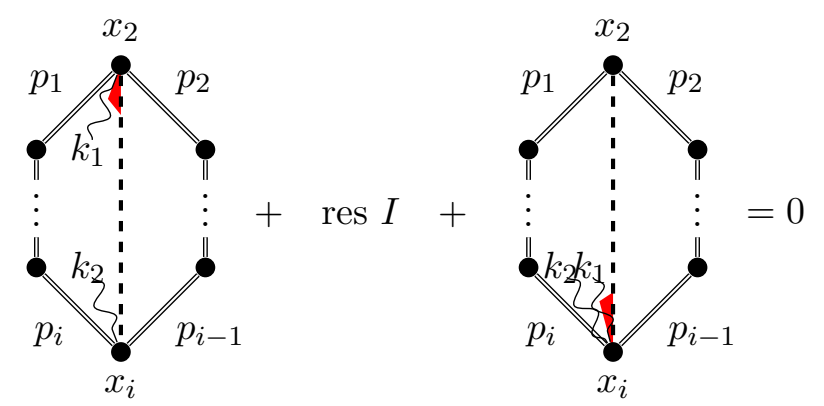

Also note that since we are simply adding zero to the sum of Feynman diagrams, to obtain a manifestly gauge invariant integrand, this means the original sum of Feynman diagrams, as integrals in Minkowski space is gauge invariant as expected, despite appearances. Since the complex spurious poles cancels in the gauge invariant integrand, we are now allowed to Wick rotate it and perform the Fourier transform in Euclidean space. At this point we use the simple Euclidean integration procedure given by eq. (C.4), essentially replacing $q$ everywhere in the integrand with the corresponding term multiplying $q$ in the exponent. Amusingly after doing this the term $I$ is no longer vanishing, but gives a non-vanishing result and furthermore, as we explain in the following section, it is identified directly to the corresponding edge terms of the dual diagram.

As a final comment we emphasise that the addition of $I$ can also be viewed as simply a neat trick done in order to perform the Fourier transform of the $W_{n, m}^{(1,0)}$ sector: the sector is gauge invariant, and in principle the Fourier transform could be performed directly in Minkowski space. However by adding $I$ and thus removing spurious poles at the level of the integrand we are able to Wick rotate and make use of the simple Euclidean Fourier transform (C.4). The final result should be the same of course whichever method is used to compute it.

\subsection{Summary of the duality mechanism}

We are now in a position to pull everything together and prove the duality $W_{n, m}^{(1,0)}=W_{m, n}^{(0,1)}$, eq. (2.31). Having shown the $\xi$-independence of $W_{n, m}^{(1,0)}$ (by including integrals similar to the $I$ in (5.8) above, obtained from edge diagrams of $W_{m, n}^{(0,1)}$ ) we can now Wick rotate all the contributing diagrams (since there are no remaining complex poles to obstruct this) and perform the Euclidean Fourier transform according to eq. (C.4). We then recognise that cusp diagrams are directly equivalent to their graph duals, just as for the MHV sector (e.g. (5.1) equates to (5.2) after multiplication of the appropriate Parke-Taylor factors). Then there are edge diagrams of the $W_{n, m}^{(1,0)}$ sector (such as (5.3)) which have no dual in the $W_{m, n}^{(0,1)}$ sector, but which in fact give zero after performing the Euclidean Fourier transform. Thus the duality still holds for these diagrams. Finally there are non-vanishing edge diagrams of the $W_{m, n}^{(0,1)}$ sector, such as (5.4). Although these have no dual diagrams in the $W_{n, m}^{(1,0)}$ sector, we find it necessary to add equivalent terms to this sector (e.g. the expression $I$ in (5.8)) in order to ensure the absence of spurious poles at the level of the 
integrand. As explained above this addition does not affect the Wilson loop form factor since the integral vanishes in Minkowski space. However it is crucial for ensuring we have an integrand without spurious (complex) poles and hence can safely Wick rotate. Then we find that the Euclidean space integrals, $I$, are equal (up to multiplication by the appropriate Parke-Taylor factors) to the dual edge diagrams contributing to $W_{m, n}^{(0,1)}$.

In summary then at the Grassmann level $\kappa+\sigma=1$ the duality works as follows:

\begin{tabular}{|cccc|}
\hline $\begin{array}{c}W^{(1,0)} \text { sector } \\
\text { (Minkowski) }\end{array}$ & Wick & $\begin{array}{c}W^{(0,1)} \text { sector } \\
\text { (Euclid) }\end{array}$ \\
\hline cusp diagrams & $\longleftrightarrow$ & cusp diagrams \\
edge diagrams & $\longleftrightarrow$ & 0 \\
added terms $I=0$ & $\longleftrightarrow$ & edge diagrams \\
\hline
\end{tabular}

\section{Concluding remarks}

In this paper we have given a proof of a new duality for Wilson loop form factors at the first non-trivial NMHV-like level and in the Born approximation. Can we go beyond?

Consider the general duality (2.32) in the Born approximation. In this case the cusp diagrams involve several propagators (see figure 1) and the corresponding edge diagrams also have a more complicated structure. In particular we need diagrams involving higherorder edge terms in the expansion of the Wilson lines $E_{i+1, i}$, eq. (3.10). Also, we encounter diagrams of the mixed type, with cups-to-cusp and cusp-to-edge propagators. Nevertheless the mechanism of spurious pole cancellation is expected to be essentially the same.

We can start with the cusp diagrams for which the duality is evident since it is a duality of planar graphs. These diagrams provide the physical poles corresponding to vanishing invariant masses, $\left(k_{i}+\cdots+k_{j-1}\right)^{2}=y_{i j}^{2}=0$, or to the distance between two distant points of the Wilson loop contour becoming lightlike, $x_{i j}^{2}=0$. However they contain various complex spurious poles. These poles are removed by adding the appropriate mixed and edge diagrams. For each spurious pole there are correction terms obtained by sliding an external leg along a propagator. The mechanism is expected to work iteratively, first removing the poles of the pure cusp diagrams, then of the mixed, etc.

We can also think of the duality beyond the Born approximation. The loop corrections to the vacuum expectation value of the Wilson loop create UV-divergences. At loop level the scattering amplitude suffers from IR-divergences. Since the Wilson loop form factor is a hybrid observable interpolating between the two, its perturbative corrections are both UV- and IR-divergent. ${ }^{7}$ So one needs to introduce a regularisation which can handle both types of divergences. Instead, we can consider the duality for the four-dimensional loop integrands corresponding to Lagrangian insertions into the Born-level object.

In the planar limit the loop integrands are unambiguously well-defined rational functions. So it is natural to expect that the duality works for them similarly to the Born

\footnotetext{
${ }^{7}$ Notice that the divergent part of the Wilson loop form factor automatically satisfies the duality relation (2.12). Namely, the IR divergencies of $W_{n, m}$ match the UV divergences of $W_{m, n}$ and vice versa.
} 


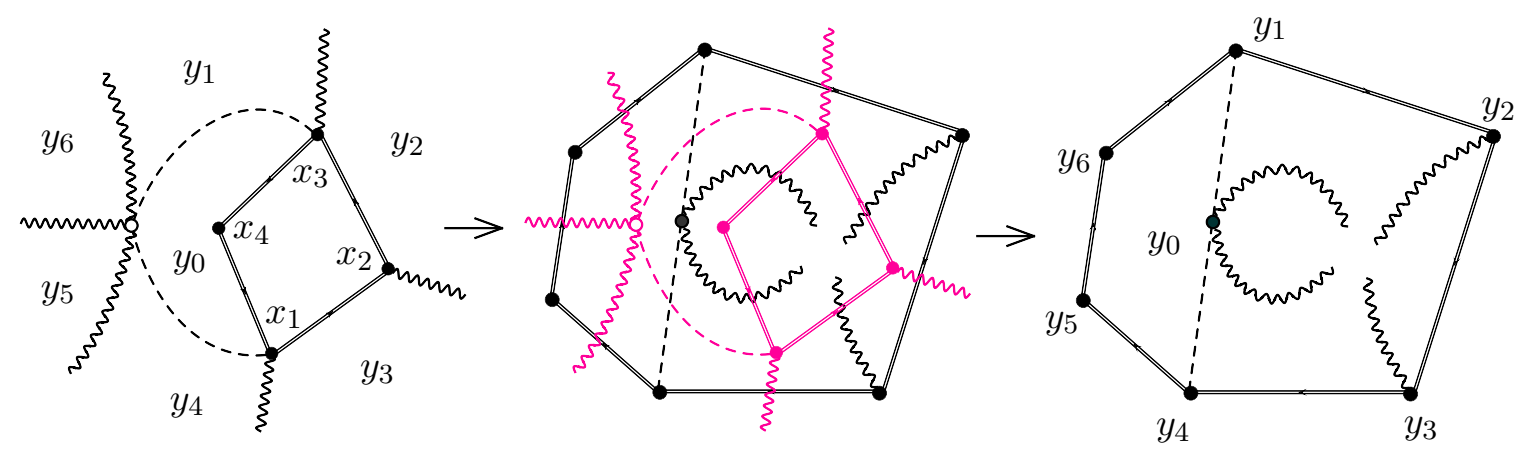

Figure 4. Diagrammatic representation of the duality relation $W_{4,6}^{(0,0)} \leftrightarrow W_{6,4}^{(0,0)}$ in the one-loop approximation.

approximation. Indeed, using the effective Feynman rules of appendix B.2 together with the Euclidean Fourier integration rules ${ }^{8}$ of appendix $\mathrm{C}$ one can see that the cusp diagrams are dual to each other as loop integrands. The corresponding edge diagrams play an auxiliary role cancelling spurious complex poles. The duality (2.12) is again translated into a planar graph duality. In figure 4 we give an example of the duality in the $\mathrm{MHV} \times \mathrm{MHV}$ sector in the one-loop approximation. There the Wilson loop contour is purely bosonic and the scattered particles are $(+1)$ helicity gluons (this is equivalent to explicitly performing the integration over the superspace variable related to point $y_{0}$ which the effective rules naturally give us). In the left diagram we introduce the region momenta $y_{0}, y_{1}, \ldots, y_{6}$ associated with faces and represent the momentum space integral as an integration over $y_{0}$. Multiplying it by the Parke-Taylor prefactor we obtain the contribution to $W_{4,6}^{(0,0)}$,

$$
\begin{aligned}
\int d^{4} y_{0} \frac{e^{i x_{23} y_{2}} e^{i x_{12} y_{3}} e^{i y_{0} x_{31}}\left[\xi\left|y_{10} y_{04}\right| \xi\right]^{3}}{y_{10}^{2} y_{04}^{2}\left[\xi\left|y_{04}\right| k_{4}\right\rangle\left\langle k_{6}\left|y_{10}\right| \xi\right]\left\langle p_{3}\left|y_{10}\right| \xi\right]\left[\xi\left|y_{10}\right| k_{1}\right\rangle\left\langle k_{3}\left|y_{04}\right| \xi\right]\left[\xi\left|y_{04}\right| p_{4}\right\rangle} \\
\times \frac{\left\langle p_{4} p_{1}\right\rangle\left\langle p_{1} p_{2}\right\rangle\left\langle p_{2} p_{3}\right\rangle\left\langle k_{6} k_{1}\right\rangle\left\langle k_{1} k_{2}\right\rangle\left\langle k_{2} k_{3}\right\rangle\left\langle k_{3} k_{4}\right\rangle}{\left\langle k_{1} p_{2}\right\rangle\left\langle p_{2} k_{2}\right\rangle\left\langle k_{2} p_{1}\right\rangle\left\langle p_{1} k_{3}\right\rangle}
\end{aligned}
$$

In the right diagram we use the Euclidean Fourier transform to write it down immediately in coordinate space and integrate over position $y_{0}$ of the interaction vertex. Its contribution to $W_{6,4}^{(0,0)}$ coincides with (6.1). So we see the duality at the level of the integrand.

There are several directions for further investigations. It is well known that the Bornlevel amplitudes have a remarkable dual superconformal symmetry which, combined with the native superconformal symmetry, results in a Yangian structure [31, 35-37]. As a result, the form of the amplitude is completely determined by this powerful symmetry and the requirement of absence of spurious poles. In this context we may ask the question if the new duality found in this paper could be a manifestation of some hidden symmetry? The first step in this direction should be to elucidate the role of conformal symmetry. It is supposed to simultaneously act on the Wilson loop component of the form factor as a local symmetry, and on its amplitude component as a non-local symmetry. This issue is under investigation.

\footnotetext{
${ }^{8}$ We cannot fully justify applicability of the Fourier transform in Euclidean space until we have checked for integrand level cancellation of spurious poles, but we assume this here.
} 
It would also be interesting to understand how to properly regularise the loop correction integrals so that the duality still holds at loop level. Another challenging problem is to find a strong coupling or AdS/CFT analog of this duality.

\section{Acknowledgments}

We profited from numerous discussions with Simon Caron-Huot and Ömer Gürdoğan. G.K. would like to thank Ömer Gürdoğan for collaboration at the early stage of this project. We acknowledge partial support by the French National Agency for Research (ANR) under contract StrongInt (BLANC-SIMI-4-2011). The work of D.C. has been partially supported by the RFBR grant 14-01-00341. The work of P.H. has been partially supported by an STFC Consolidated Grant ST/L000407/1. P.H. would also like to thank the CNRS for financial support and LAPTh for hospitality where part of this work was done.

\section{A Chiral lightlike Wilson loop in LHC superspace}

\section{A.1 Chiral lightlike Wilson loop}

The conventional formulation of a chiral supersymmetric Wilson line on a segment of the line in $(x, \theta)$-superspace

$$
x_{\alpha \dot{\alpha}}(t)=x_{\alpha \dot{\alpha}}-t p_{\alpha} \tilde{p}_{\dot{\alpha}}, \quad \theta_{\alpha}^{A}(t)=\theta_{\alpha}^{A}-t \omega^{A} p_{\alpha}, \quad t \in[0,1],
$$

has the form (recall $(2.27))$

$$
\mathcal{E}=P \exp \left\{-i \int_{0}^{1} d t\left[\frac{1}{2} \tilde{p}^{\dot{\alpha}} p^{\alpha} \mathcal{A}_{\alpha \dot{\alpha}}(x(t), \theta(t))+\omega^{A} p^{\alpha} \mathcal{A}_{\alpha A}(x(t), \theta(t))\right]\right\}
$$

The corresponding covariant derivatives $\mathcal{D}=\partial+\mathcal{A}$ transform under a gauge group with ordinary, harmonic-independent parameters,

$$
\mathcal{D} \rightarrow e^{\tau(x, \theta)} \mathcal{D} e^{-\tau(x, \theta)} .
$$

Consequently the Wilson line transforms as follows:

$$
\mathcal{E} \rightarrow e^{\tau(x(1), \theta(1))} \mathcal{E} e^{-\tau(x(0), \theta(0))} .
$$

A complete gauge-invariant lightlike Wilson loop is obtained by gluing together $n$ consecutive segments and closing the contour (with the identification $n+1 \equiv 1$ ),

$$
\mathcal{W}_{n}=\frac{1}{N} \operatorname{tr} \prod_{i=1}^{n} \mathcal{E}_{i+1, i}
$$




\section{A.2 Bridge transformation to LHC superspace}

For our purposes we need to express the Wilson loop (A.5) in terms of the unconstrained prepotentials $A^{++}, A_{\dot{\alpha}}^{+}$from (3.1). To this end we need to relate the covariant derivatives $\mathcal{D}$ with the transformation (A.3) to the derivatives $\nabla$ with the transformation (3.3). The key observation is that the harmonic derivative $\partial^{++}$needs no connection for the gauge group with harmonic-independent parameters $\tau(x, \theta), \mathcal{D}^{++}=\partial^{++}$. This suggests to relate it to $\nabla^{++}$from (3.2) by a generalised gauge transformation,

$$
\partial^{++}=h^{-1} \nabla^{++} h=h^{-1}\left(\partial^{++}+A^{++}\right) h
$$

or equivalently

$$
A^{++}\left(x, \theta^{+}, u\right)=-\left(\partial^{++} h\right) h^{-1}=h \partial^{++} h^{-1} .
$$

Here the 'parameter' $h(x, \theta, u)$ depends on all the LHC superspace variables. It undergoes gauge transformations under both gauge groups,

$$
h \rightarrow e^{\Lambda\left(x, \theta^{+}, u\right)} h e^{-\tau(x, \theta)} .
$$

In the Abelian case this becomes $\delta h(x, \theta, u)=\Lambda\left(x, \theta^{+}, u\right)-\tau(x, \theta)$ and we see that the combined gauge transformations of both types cannot gauge away the entire content of the general superfield $h$. Hence, despite the appearance $h$ is not a pure gauge. In the same way, (A.6) and (A.7) are not gauge transformations but rather field redefinitions. We call the new object $h$ a 'gauge bridge ${ }^{9}$ relating the $\tau$-frame with harmonic-independent parameters and the $\Lambda$-frame with analytic parameters.

Relation (A.7) is a differential equation on the harmonic sphere $S^{2}$. In it we consider the chiral-analytic prepotential $A^{++}$as given and the bridge $h$ as the unknown. This equation has a solution defined up to arbitrary $\tau$ and $\Lambda$ gauge transformations, so the bridge $h$ cannot be obtained unambiguously from the prepotential.

Once the bridge has been found, it can be used to convert any gauge covariant object from the $\tau$-frame to the $\Lambda$-frame or vice versa. In particular, all covariant derivatives $\mathcal{D}$ can be converted to $\nabla$,

$$
\nabla=h \mathcal{D} h^{-1}
$$

transforming according to (3.3). Then we can do the same with the Wilson line (A.2):

$$
E=h(x(1), \theta(1), u) \mathcal{E} h^{-1}(x(0), \theta(0), u)
$$

with the gauge transformation

$$
E \rightarrow e^{\Lambda\left(x(1), \theta^{+}(1), u\right)} E e^{-\Lambda\left(x(0), \theta^{+}(0), u\right)} .
$$

Note that we use the same harmonic variable $u$ all along the segment.

Conversely, starting from the $\Lambda$-frame Wilson line and transforming it back to the $\tau$-frame, we obtain an object which does not depend on the harmonics:

$$
\mathcal{E}=h^{-1}(x(1), \theta(1), u) E(u) h(x(0), \theta(0), u), \quad \partial^{++} \mathcal{E}=0 .
$$

\footnotetext{
${ }^{9}$ The terminology originates from the harmonic superspace formulation of $\mathcal{N}=2 \mathrm{SYM}[38]$. A similar object exists in the Ward construction for self-dual non-supersymmetric Yang-Mills [39, 40].
} 
Indeed, the harmonic dependence of the $\Lambda$-frame Wilson line (A.10) comes from the bridge $h$. The inverse transformation (A.12) removes the bridge and with it the $u$-dependence. This property allows us to choose the harmonics $u$ on a given segment as we like. A judicious choice $[5,26]$ is to identify the harmonic $u^{+}$with the chiral spinor defining the direction of the lightlike line,

$$
u_{\alpha}^{+} \equiv p_{\alpha}
$$

and $u^{-}$with the $\mathrm{SU}(2)$ conjugate $\bar{p}_{\alpha}$. With this choice we obtain (see (A.1))

$$
\begin{aligned}
E & =P \exp \left\{-i \int_{0}^{1} d t\left[\frac{1}{2} \tilde{p}^{\dot{\alpha}} p^{\alpha} A_{\alpha \dot{\alpha}}(x(t), \theta(t),|p\rangle)+\omega^{A} p^{\alpha} A_{\alpha A}(x(t), \theta(t),|p\rangle)\right]\right\} \\
& =P \exp \left\{-i \int_{0}^{1} d t\left[\frac{1}{2} \tilde{p}^{\dot{\alpha}} A_{\dot{\alpha}}^{+}(x(t), \theta(t),|p\rangle)+\omega^{A} A_{A}^{+}(x(t), \theta(t),|p\rangle)\right]\right\} \\
& =P \exp \left\{-\frac{i}{2} \int_{0}^{1} d t \tilde{p}^{\dot{\alpha}} A_{\dot{\alpha}}^{+}(x(t),\langle p \theta\rangle,|p\rangle)\right\} .
\end{aligned}
$$

In the last relation we have used the $\Lambda$-frame property $A_{A}^{+}=0$, i.e. $\nabla_{A}^{+}=\partial_{A}^{+}$. The gauge connection contributing to the Wilson line is the prepotential $A_{\dot{\alpha}}^{+}\left(x, \theta^{+}, u\right)$ from (3.1) with $\theta^{+A}=u^{+\alpha} \theta_{\alpha}^{A}(t)=p^{\alpha}\left(\theta_{\alpha}^{A}-t \omega^{A} p_{\alpha}\right)=\left\langle p \theta^{A}\right\rangle$. So, the only dependence on the position along the lightlike segment is in the space-time coordinate $x(t)$.

Let us now glue together the different segments of a Wilson loop according to (A.5). Consider two adjacent segments:

$$
\begin{aligned}
& \mathcal{E}_{i, i-1}=h^{-1}\left(x_{i}, \theta_{i}, p_{i-1}\right) E_{i, i-1} h\left(x_{i-1}, \theta_{i-1}, p_{i-1}\right) \\
& \mathcal{E}_{i+1, i}=h^{-1}\left(x_{i+1}, \theta_{i+1}, p_{i}\right) E_{i+1, i} h\left(x_{i}, \theta_{i}, p_{i}\right) .
\end{aligned}
$$

In their product $\mathcal{E}_{i+1, i} \mathcal{E}_{i, i-1}$ the bridges at the cusp point $i$ depend on the same superspace coordinates $x_{i}, \theta_{i}$ but on different harmonics $p_{i-1}$ and $p_{i}$. The two adjacent bridges define a new object,

$$
h\left(x_{i}, \theta_{i}, p_{i}\right) h^{-1}\left(x_{i}, \theta_{i}, p_{i-1}\right):=U\left(x_{i}, \theta_{i} ; p_{i}, p_{i-1}\right) .
$$

This object has been discussed in detail in [30]. The bridge $h(x, \theta, u)$ relates the harmonic-independent $\tau$ - gauge frame and the analytic $\Lambda$-gauge frame. The new bridge

$$
U(x, \theta ; u, v)=h(x, \theta, u) h^{-1}(x, \theta, v)
$$

is inert under the $\tau$-frame gauge transformations but transforms with respect to two analytic frames with harmonics $v$ and $u$ (see (A.8)),

$$
U(x, \theta ; u, v) \rightarrow e^{\Lambda\left(x, \theta^{+}, u\right)} U(x, \theta ; u, v) e^{-\Lambda\left(x, \theta^{+}, v\right)} .
$$

We call this object a bilocal bridge, where the biloclality refers only to the harmonic variables. An explicit expression for $U$ in terms of the prepotential $A^{++}$from (3.1) can be found by solving the harmonic differential equation that follows from (A.17) and from the definition (A.6) of the bridge $h$,

$$
\nabla_{u}^{++} U(u, v)=0
$$

with the obvious boundary condition $U(u, u)=1$. The solution [41] is given in (3.9). 
In conclusion, coming back to the closed Wilson loop (A.5), we obtain the formulation of the chiral lightlike Wilson loop in LHC superspace by gluing together Wilson line segments (A.14) and using bilocal bridges (A.17) as 'glue', eq. (3.8), where $E_{i+1, i}$ is defined in (3.10). Comparing the gauge transformations of the bilocal bridge $U$ (A.18) and of the Wilson line segments $E$ (A.11), we clearly see the role of the bridges: they adjust the transformation properties of the segments at the cusp points.

\section{A.3 Comments on the twistor formulation of Mason and Skinner}

The construction of lightlike Wilson loops presented here is similar in spirit to the twistor formulation of Mason and Skinner in [5]. After establishing the LHC/twistor dictionary (see [23]), we can make a detailed comparison.

The noticeable difference concerns the claim in [5] that the entire Wilson loop can be written as a product of 'parallel propagators' $U$ [5, 24, 42],

$$
\mathcal{W}_{n}^{\mathrm{M} \& \mathrm{~S}}=\frac{1}{N} \operatorname{tr} \prod_{i=1}^{n} U\left(x_{i}, \theta_{i} ; p_{i}, p_{i-1}\right) .
$$

Equation (6.16) (see also eq. (2.17)) in [5] displays this form of the Wilson loop. Comparing with our formulation (3.8), we see that the Wilson line edge factors $E_{i+1, i}$ are missing in (A.20). One would wonder how such an incomplete expression can be gauge invariant? Indeed, from (A.18) we see that the product $U\left(x_{i+1}, \theta_{i+1}, p_{i+1}, p_{i}\right) U\left(x_{i}, \theta_{i}, p_{i}, p_{i-1}\right)$ is not gauge invariant at point $i$; the role of the edge factor $E_{i+1, i}$ is precisely to adjust the transformation properties.

The attentive reader may notice that on each segment of the Wilson loop Mason and Skinner impose the condition $\tilde{p}^{\dot{\alpha}} A_{\dot{\alpha}}^{+}=0$ (in our notation; see the text before their equation (6.14)). This condition would indeed trivialise (3.10) but it is not clear where it comes from. It looks like a 'floating gauge condition', i.e. the light-cone gauge $\xi^{\dot{\alpha}} A_{\dot{\alpha}}^{+}=0$ (B.1) but with $\xi \equiv \tilde{p}_{i}$ for each segment. In our understanding, if we change the gauge-fixing condition from one segment of the Wilson loop to the other, we would break gauge invariance. The edge contribution $E_{i+1, i}$ in (3.8) is an indispensable part of the definition of the Wilson loop. In section 5 we have shown explicit examples where the edges contribute essential pieces of the complete NMHV Wilson loops. In fact, they are responsible for obtaining a gauge-invariant, i.e. $\xi$-independent result.

\section{B Feynman rules}

\section{B.1 Propagators and on-shell states}

To quantise the theory we use the light-cone (or axial or CSW) gauge [25, 43]

$$
\xi^{\dot{\alpha}} A_{\dot{\alpha}}^{+}=0,
$$

where the gauge-fixing parameter $\xi^{\dot{\alpha}}$ is an antichiral commuting spinor ("reference spinor"). This gauge has the advantage that the cubic Chern-Simons term in (3.5) vanishes, so the interaction comes only from $L_{Z}$ (3.6). However, it introduces specific spurious poles in the 
propagators which require careful treatment. We find that the correct way is to implement all Feynman rules in momentum space, even though the Wilson loop is defined in position space. The Fourier integrals from position to momentum space are initially defined in Minkowski space. Only after we have made sure that the sum of all Feynman diagrams is free from spurious poles, we can Wick rotate to Euclidean space and evaluate the Fourier integrals by the simple formulas in appendix $\mathrm{C}$.

The Feynman rules in the gauge (B.1) have been worked out in [23] in position space and their momentum space equivalents in [30]. Here we summarise the effective rules after the Grassmann and harmonic integrations at the vertices have been carried out.

The propagators are determined from the (non-diagonal) quadratic part of the ChernSimons Lagrangian (3.5): ${ }^{10}$

$$
\begin{aligned}
\left\langle A^{++}\left(q, \theta_{1}^{+}, u_{1}\right) A^{++}\left(-q, \theta_{2}^{+}, u_{2}\right)\right\rangle & =4 \pi \delta^{2}\left(\left\langle u_{1}^{+}|q| \xi\right]\right) \delta\left(u_{1}, u_{2}\right) \delta^{4}\left(\left\langle u_{1}^{+} \theta_{12}\right\rangle\right), \\
\left\langle A_{\dot{\alpha}}^{+}\left(q, \theta_{1}^{+}, u_{1}\right) A^{++}\left(-q, \theta_{2}^{+}, u_{2}\right)\right\rangle & =\frac{4 \mathrm{i} \xi_{\dot{\alpha}}}{\left\langle u_{1}^{+}|q| \xi\right]} \delta\left(u_{1}, u_{2}\right) \delta^{4}\left(\left\langle u_{1}^{+} \theta_{12}\right\rangle\right)
\end{aligned}
$$

and $\left\langle A_{\dot{\alpha}}^{+} A_{\dot{\beta}}^{+}\right\rangle=0$. The harmonics are auxiliary variables which are integrated out in gaugeinvariant quantities. The harmonic integrations originate from the interaction vertices in the Lagrangian $L_{Z}$ (3.6) and in the bilocal bridge $U$ (3.9). They are implemented with the help of delta functions. Firstly, the harmonic delta-function $\delta\left(u_{1}, u_{2}\right)$ identifies $u_{1}^{ \pm}=u_{2}^{ \pm}$, so each line effectively carries only one harmonic. Secondly, the remaining harmonic of the propagator $\left\langle A^{++} A^{++}\right\rangle$is integrated out by means of the delta-function $\delta^{2}$, eq. (B.2), resulting in the substitution $\left.u^{+} \rightarrow q \mid \xi\right]$ and the Jacobian factor $1 / q^{2}$, i.e.

$$
\left.\int d u \delta^{2}\left(\left\langle u^{+}|q| \xi\right]\right) \mathcal{R}(u)=\frac{1}{\pi q^{2}} \mathcal{R}(q \mid \xi]\right),
$$

for a homogeneous function of degree zero $\mathcal{R}(u)$. In the effective Feynman rules all harmonic integrations are already implemented.

Another effective Feynman rule concerns the emission of $n$ external states and of one propagator from an interaction vertex in the Lagrangian $L_{Z}$. Denoting by $K=\sum_{1}^{n} k_{i}$ and $Q_{K}=\sum_{1}^{n}\left|k_{i}\right\rangle \eta_{i}$ the total (super)momentum of the $n$ particles and using the Feynman rules (B.2) we find:

$$
\begin{aligned}
& \int d^{4} x_{0} d^{8} \theta_{0}\left\langle\left(k_{1}, \eta_{1}\right) \ldots\left(k_{n}, \eta_{n}\right)\left|L_{Z}\left(x_{0}, \theta_{0}\right) A^{++}\left(x, \theta^{+}, u\right)\right| 0\right\rangle \\
& =\int d^{4} x_{0} d^{8} \theta_{0} e^{i K x_{0}+Q_{K} \theta_{0}} \int \frac{d^{4} q}{(2 \pi)^{4}} \frac{e^{i q\left(x_{0}-x\right)}}{q^{2}} \delta^{4}\left(\left\langle\theta_{0}-\theta|q| \xi\right]\right) \frac{1}{\left[\xi|q| k_{1}\right\rangle\left\langle k_{1} k_{2}\right\rangle \ldots\left\langle k_{n}|q| \xi\right]} \\
& =\frac{e^{i K x+Q_{K} \theta}}{K^{2}} \frac{\delta^{4}\left(\left\langle Q_{K}|K| \xi\right]\right)}{\left[\xi|K| k_{1}\right\rangle\left\langle k_{1} k_{2}\right\rangle \ldots\left\langle k_{n}|K| \xi\right]} .
\end{aligned}
$$

The prepotential $A^{++}$plays the leading role. The diagrams with propagators $\left\langle A^{++} A^{++}\right\rangle$provide the main contribution (cusp diagrams). The prepotential $A_{\dot{\alpha}}^{+}$and the propagator $\left\langle A^{++} A_{\dot{\alpha}}^{+}\right\rangle$appear in edge diagrams which are needed to restore gauge invariance (i.e., to eliminate the reference spinor $\xi$ and its spurious poles). The only dependence

\footnotetext{
${ }^{10}$ The bilinear term in $L_{Z}$ is treated as a bivalent vertex.
} 
on $A_{\dot{\alpha}}^{+}$comes from the edges $E_{i+1, i}$ of the Wilson loop. The prepotential $A_{\dot{\alpha}}^{+}$slides along an edge of the Wilson loop contour, see (3.10). At NMHV level we deal with the linear approximation of the Pexp. The corresponding line integral concerns only the exponential factors (Fourier or wave function), e.g. $\int_{0}^{1} d t e^{i q\left(x_{i}-t p_{i}\right)}=\frac{1}{i\left(q p_{i}\right)}\left(e^{i q x_{i}}-e^{i q x_{i+1}}\right)$.

In the following we deal mostly with harmonics $u^{+}$carrying $\mathrm{U}(1)$-charge $(+1)$, so we omit the + index of the harmonics and the Grassmann variables for the sake of brevity.

\section{B.2 Effective Feynman rules}

To obtain effective Feynman rules we carry out all harmonic and Grassmann integrations.

\section{B.2.1 Vertices}

The bilocal bridge $U\left(x, \theta ; p_{1}, p_{2}\right)$, eq. (3.9), at the lowest order in the coupling equals 1 :

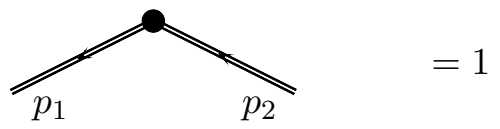

Higher-order vertices in the expansion of $U\left(x, \theta ; p_{2}, p_{1}\right)$. In this example the line with $u_{2}$ ends on a superstate $(k, \eta)$ and depicts particle emission. The harmonic is identified with the particle momentum, $u_{2} \rightarrow|k\rangle$. The other lines are internal. If an internal line depicts the propagator $\left\langle A^{++}(q, u) A^{++}(-q, u)\right\rangle$, its harmonic is substituted by $\left.u^{+} \rightarrow q \mid \xi\right]$, see eq. (B.4). If an internal line depicts the propagator $\left\langle A^{++} A_{\dot{\alpha}}^{+}\right\rangle$, then $u^{+}$is identified with the lightlike direction spinor $|p\rangle$ of the edge where the prepotential $A_{\dot{\alpha}}^{+}$lives.

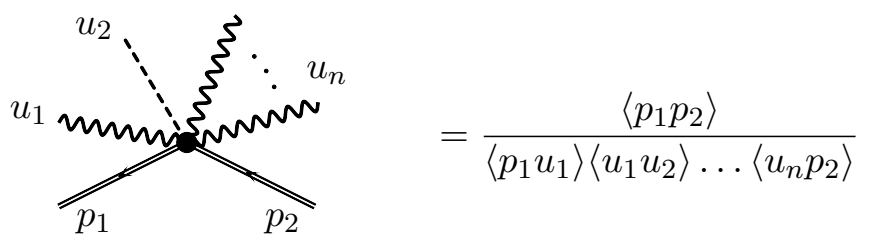

Interaction vertex $L_{Z}$, eq. (3.6). Lines $i=1,3$ correspond to particle emission. The other lines are internal. The harmonics are identified as above.

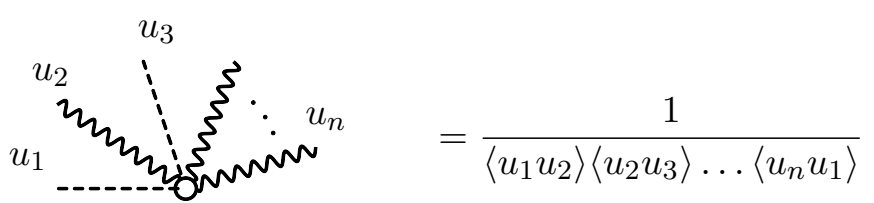

\section{B.2.2 Propagators}

1. Propagator $\left\langle A^{++} A^{++}\right\rangle$between two Wilson loop cusps. The harmonic of the vertices (B.7) and (B.8) where this propagator ends is replaced by $\left.u^{+} \rightarrow q \mid \xi\right]$.

$$
\left.\left.\left(x_{1}, \theta_{1}, q \mid \xi\right]\right) \quad\left(x_{2}, \theta_{2}, q \mid \xi\right]\right)=\int \frac{d^{4} q}{4 \pi^{2}} e^{i q x_{12}} \frac{1}{q^{2}} \delta^{4}\left(\left\langle\theta_{12}|q| \xi\right]\right)
$$

2. Emission of a superparticle with (super)momentum $\left(k, Q_{k} \equiv \eta|k\rangle\right)$ from a cusp of the Wilson loop. The wave function $\left\langle k, Q_{k}\right| A^{++}(x, \theta,|k\rangle)|0\rangle$ is

$$
\sim^{\left(k, Q_{k},|k\rangle\right)} \sim^{(x, \theta,|k\rangle)}=e^{i k x+Q_{k} \theta}
$$


3. Emission of a superparticle from an interaction vertex $L_{Z}$.

$$
\sim^{\left(k, Q_{k},|k\rangle\right)} \sim \circ=1
$$

4. Propagator $\left\langle A^{++} A^{++}\right\rangle$between an interaction vertex and a cusp $(x, \theta)$. The momentum $K$ and supercharge $Q_{K}$ flow into this line from the side of the vertex.

$$
\left.\left.\left.\left(K, Q_{K}, K \mid \xi\right]\right)(x, \theta, K \mid \xi]\right)\right)=\frac{1}{K^{2}} e^{i K x+Q_{K} \theta} \delta^{4}\left(\left\langle Q_{K}|K| \xi\right]\right)
$$

5. Interaction vertex connected with a Wilson line segment $W_{12}$ by a propagator $\left\langle A^{++} A_{\dot{\alpha}}^{+}\right\rangle$sliding along the edge parametrised by $x(t)=x_{1}-t|p\rangle\left[p \mid, p \equiv x_{1}-x_{2}\right.$, $t \in[0,1]$. The (super)momentum $\left(K, Q_{K}\right)$ flows into this line from the side of the vertex.

$$
\left(x_{2}, \theta_{2},|p\rangle\right)
$$

6. Cusp $\left(x_{0}, \theta_{0}\right)$ connected with $W_{12}$ by a propagator sliding along the edge. Note that $\left\langle p \theta_{10}\right\rangle=\left\langle p \theta_{20}\right\rangle$.

$$
\left(x_{0}, \theta_{0},|p\rangle\right)
$$

\section{Fourier transforms}

In this paper we encounter Fourier integrals of the type

$$
\int d^{4} q \frac{e^{i q x}}{q^{2}} \mathcal{R}([\xi \mid q),
$$

where $\mathcal{R}([\xi \mid q)$ is a homogeneous function of degree zero of the spinor $[\xi \mid q$. The way this integral is computed heavily depends on the space-time signature. In Euclidean space we can use the following trick. First, we insert a harmonic integral over $S^{2}$ and write

$$
\int d^{4} q \frac{e^{i q x}}{q^{2}} \mathcal{R}\left([\xi \mid q)=\pi \int d^{4} q e^{i q x} \int d u \delta^{2}\left(\left[\xi|q| u^{+}\right\rangle\right) \mathcal{R}\left(u^{+}\right) .\right.
$$


The role of the complex delta function is to identify the $\mathrm{SU}(2)_{L}$ harmonic $\left.u^{+} \sim q \mid \xi\right]$, up to a phase factor which drops out in the homogeneous function $\mathcal{R}\left(u^{+}\right)$. The factor $1 / q^{2}$ is the corresponding Jacobian (for detail see appendix A in [23]). Then we decompose the dot product

$$
2(q \cdot x)=\left[\bar{\xi}|q| u^{+}\right\rangle\left[\xi|x| u^{-}\right\rangle+\left[\xi|q| u^{-}\right\rangle\left[\bar{\xi}|x| u^{+}\right\rangle-\left[\bar{\xi}|q| u^{-}\right\rangle\left[\xi|x| u^{+}\right\rangle-\left[\xi|q| u^{+}\right\rangle\left[\bar{\xi}|x| u^{-}\right\rangle
$$

in the basis formed by the $\mathrm{SU}(2)_{L}$ harmonics $u_{\alpha}^{ \pm}$and their $\mathrm{SU}(2)_{R}$ analogs $\xi_{\dot{\alpha}}, \bar{\xi}_{\dot{\alpha}}$ with $\left\langle u^{+} u^{-}\right\rangle=1$ and $[\xi \bar{\xi}]=1$. Next we swap the harmonic and Fourier integrals and use the delta function to lift the integral over the complex variable $\left[\xi|q| u^{+}\right\rangle$(and its conjugate $\left.\left[\bar{\xi}|q| u^{-}\right\rangle\right)$. The remaining Fourier integral over $\left[\xi|q| u^{-}\right\rangle$produces another delta function, so

$$
\int d^{4} q e^{i q x} \delta^{2}\left(\left[\xi|q| u^{+}\right\rangle\right)=4 \pi^{2} \delta^{2}\left(\left[\bar{\xi}|x| u^{+}\right\rangle\right) .
$$

Finally, we do the harmonic integral with the help of the new delta function and obtain

$$
\int \frac{d^{4} q}{4 \pi^{2}} \frac{e^{i q x}}{q^{2}} \mathcal{R}\left([\xi \mid q)=\frac{1}{x^{2}} \mathcal{R}([\bar{\xi} \mid x)\right.
$$

As an example, consider the integral

$$
\int \frac{d^{4} q}{4 \pi^{2}} \frac{e^{i q x}}{q^{2}} \frac{\left[\xi|q| p_{1}\right\rangle}{\left[\xi|q| p_{2}\right\rangle}=\frac{1}{x^{2}} \frac{\left[\bar{\xi}|x| p_{1}\right\rangle}{\left[\bar{\xi}|x| p_{2}\right\rangle}
$$

Its computation in Euclidean signature is straightforward, as shown above. However, were this integral to be evaluated with Minkowski signature, we would have to deal with the complex pole at $\left[\xi|q| p_{2}\right\rangle=0$. This is a rather nontrivial task which we need not address.

Another type of Fourier integral that we encounter is obtained by combining two integrals of the type (C.5):

$$
\begin{aligned}
\int \frac{d^{4} q}{4 \pi^{2}} e^{i q x} \frac{[\zeta \xi]}{\left[\xi|q| p_{2}\right\rangle\left[\zeta|q| p_{1}\right\rangle} & =\frac{1}{\left\langle p_{1} p_{2}\right\rangle} \int \frac{d^{4} q}{4 \pi^{2}} \frac{e^{i q x}}{q^{2}}\left[\frac{\left[\xi|q| p_{1}\right\rangle}{\left[\xi|q| p_{2}\right\rangle}-\frac{\left[\zeta|q| p_{1}\right\rangle}{\left[\zeta|q| p_{2}\right\rangle}\right] \\
& =\frac{[\bar{\zeta} \bar{\xi}]}{\left[\bar{\xi}|x| p_{2}\right\rangle\left[\bar{\zeta}|x| p_{1}\right\rangle} .
\end{aligned}
$$

We remark that in Minkowski signature, with the choice $\left[\zeta \mid=\bar{p}_{1}\right.$, this integral becomes a contact term $\sim \delta\left(x \cdot p_{1}\right)$. At the same time, in Euclidean signature it is a rational function. This example clearly illustrates the drastic difference between the two spacetime signatures. It also explains why we prefer to do all our Fourier integrals after the Wick rotation to Euclidean space.

\section{Cancellation of spurious poles: boundary cases}

Here we discuss in more details the cancellation of spurious poles between cusp and edge diagrams at the Grassmann level $\kappa+\sigma=1$. 
$\mathbf{M H V} \times \mathbf{N M H V}$ sector. From the discussion in section 5.3 we see that spurious poles in the $W^{(0,1)}$ sector are cancelled by nearby diagrams. In particular, in (5.5) we see that spurious poles of the type $\left[\xi\left|x_{2 i}\right| p_{2}\right\rangle=0$ are cancelled using similar diagrams with the leg $p_{2}$ shifted from the right end to the left end of the propagator or vice versa. Eventually however one will run out of free legs to shift in this way and we refer to these situations as boundary cases. The case where there are no more legs to shift to the right is covered by the edge diagram as in (5.6). We need however to consider the case when there are not enough legs on the right to shift left. More precisely we need to consider the case when there are just two legs entering the propagator from the right. Then if we were to shift the final propagator to the left this would leave a diagram with just one leg entering the propagator which vanishes. So which diagram cancels the spurious pole in this case? The answer is that the pole is canceled by a similar diagram where two legs have shifted, for example
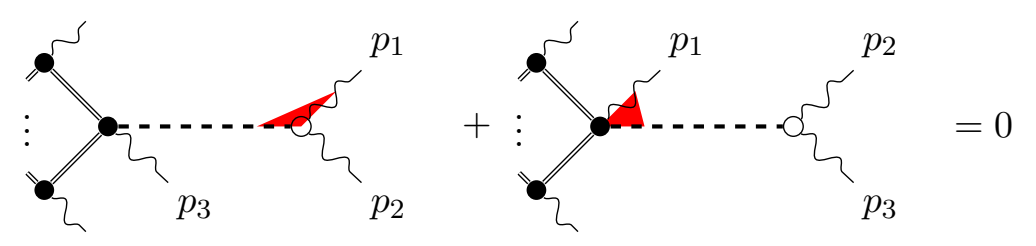

Here leg $p_{1}$ has shifted left as expected but leg $p_{3}$ has simultaneously shifted right. One can straightforwardly check that the spurious pole in question cancels between these two diagrams.

Finally then there arises the situation where there is no leg (such as $p_{3}$ in the example) to shift to the right in this way. In this final case it turns out that the spurious pole is canceled by an edge diagram with a single leg entering the propagator. Such a diagram is allowed unlike the similar vertex diagram with one leg entering the propagator.

So for example we get the mutual cancellation of the spurious poles in the following diagrams:
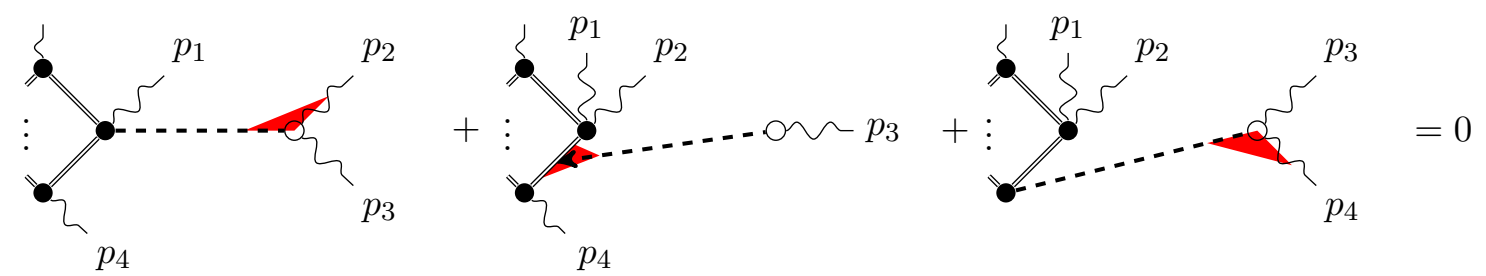

If there had been no leg $p_{4}$ in the above situation, the single legged propagator would simply continue around the Wilson loop, cancelling all spurious poles, until it did reach a leg.

We note that the edge diagram with the single leg entering it thus serves a crucial purpose in cancelling spurious poles, but that these are different types of spurious poles to the type of pole the generic edge diagrams cancel (compare the positions of the spurious poles in (D.2) with those of (5.6)).

$\mathbf{N M H V} \times \mathbf{M H V}$ sector. We also need to consider the various boundary cases of the $W^{(1,0)}$ sector. Here we need to show cancellation of spurious poles at the level of the 
integrand. Firstly consider the spurious pole cancellation illustrated in (5.7). There we see that spurious poles of the form $\left[\xi|q| p_{2}\right\rangle=0$ cancel with nearby diagrams where the propagator slides around the Wilson loop. This procedure continues unimpeded until either one end of the propagator meets a leg, or the two ends of the propagator get too close to each other. In the former case there is a remaining spurious pole with apparently no diagram to cancel it. This is precisely the case discussed in (5.9)) where one introduces the expression $I$ which cancels the spurious pole in question. In the latter case we end up with a propagator stretched between two adjacent vertices. In fact for this case there is no remaining spurious pole to cancel. This diagram only has two spurious poles (rather than four) which have already been cancelled by nearby diagrams.

So to summarise, in this sector all the boundary cases take care of themselves, following the introduction of the integrands $I$ discussed in (5.8).

Open Access. This article is distributed under the terms of the Creative Commons Attribution License (CC-BY 4.0), which permits any use, distribution and reproduction in any medium, provided the original author(s) and source are credited.

\section{References}

[1] L.F. Alday and J.M. Maldacena, Gluon scattering amplitudes at strong coupling, JHEP 06 (2007) 064 [arXiv: 0705.0303] [INSPIRE].

[2] J.M. Drummond, G.P. Korchemsky and E. Sokatchev, Conformal properties of four-gluon planar amplitudes and Wilson loops, Nucl. Phys. B 795 (2008) 385 [arXiv:0707.0243] [INSPIRE].

[3] A. Brandhuber, P. Heslop and G. Travaglini, $M H V$ amplitudes in $N=4$ super Yang-Mills and Wilson loops, Nucl. Phys. B 794 (2008) 231 [arXiv:0707.1153] [INSPIRE].

[4] S. Caron-Huot, Notes on the scattering amplitude/Wilson loop duality, JHEP 07 (2011) 058 [arXiv: 1010.1167] [INSPIRE].

[5] L.J. Mason and D. Skinner, The complete planar S-matrix of $N=4$ SYM as a Wilson loop in twistor space, JHEP 12 (2010) 018 [arXiv:1009.2225] [INSPIRE].

[6] A.V. Belitsky, G.P. Korchemsky and E. Sokatchev, Are scattering amplitudes dual to super Wilson loops?, Nucl. Phys. B 855 (2012) 333 [arXiv:1103.3008] [INSPIRE].

[7] L.F. Alday, B. Eden, G.P. Korchemsky, J. Maldacena and E. Sokatchev, From correlation functions to Wilson loops, JHEP 09 (2011) 123 [arXiv: 1007.3243] [INSPIRE].

[8] B. Eden, G.P. Korchemsky and E. Sokatchev, From correlation functions to scattering amplitudes, JHEP 12 (2011) 002 [arXiv: 1007.3246] [INSPIRE].

[9] B. Eden, P. Heslop, G.P. Korchemsky and E. Sokatchev, The super-correlator/super-amplitude duality: part I, Nucl. Phys. B 869 (2013) 329 [arXiv:1103.3714] [INSPIRE].

[10] B. Eden, P. Heslop, G.P. Korchemsky and E. Sokatchev, The super-correlator/super-amplitude duality: part II, Nucl. Phys. B 869 (2013) 378 [arXiv: 1103.4353] [INSPIRE]. 
[11] T. Adamo, M. Bullimore, L. Mason and D. Skinner, A proof of the supersymmetric correlation function/Wilson loop correspondence, JHEP 08 (2011) 076 [arXiv:1103.4119] [INSPIRE].

[12] A. Brandhuber, B. Spence, G. Travaglini and G. Yang, Form factors in $N=4$ super Yang-Mills and periodic Wilson loops, JHEP 01 (2011) 134 [arXiv:1011.1899] [INSPIRE].

[13] A. Brandhuber, O. Gurdogan, R. Mooney, G. Travaglini and G. Yang, Harmony of super form factors, JHEP 10 (2011) 046 [arXiv:1107.5067] [INSPIRE].

[14] O.T. Engelund and R. Roiban, Correlation functions of local composite operators from generalized unitarity, JHEP 03 (2013) 172 [arXiv:1209.0227] [INSPIRE].

[15] L. Koster, V. Mitev, M. Staudacher and M. Wilhelm, All tree-level MHV form factors in $N=4$ SYM from twistor space, JHEP 06 (2016) 162 [arXiv:1604.00012] [INSPIRE].

[16] L. Koster, V. Mitev, M. Staudacher and M. Wilhelm, On form factors and correlation functions in twistor space, JHEP 03 (2017) 131 [arXiv:1611.08599] [INSPIRE].

[17] L.V. Bork and A.I. Onishchenko, Grassmannians and form factors with $q^{2}=0$ in $N=4$ SYM theory, JHEP 12 (2016) 076 [arXiv:1607.00503] [INSPIRE].

[18] L.V. Bork and A.I. Onishchenko, Wilson lines, Grassmannians and gauge invariant off-shell amplitudes in $N=4$ SYM, JHEP 04 (2017) 019 [arXiv:1607.02320] [INSPIRE].

[19] S.É. Derkachov, G.P. Korchemsky and A.N. Manashov, Dual conformal symmetry on the light-cone, Nucl. Phys. B 886 (2014) 1102 [arXiv:1306.5951] [INSPIRE].

[20] S. Caron-Huot, unpublished notes.

[21] J.P. Harnad and S. Shnider, Constraints and field equations for ten-dimensional super-Yang-Mills theory, Commun. Math. Phys. 106 (1986) 183 [INSPIRE].

[22] H. Ooguri, J. Rahmfeld, H. Robins and J. Tannenhauser, Holography in superspace, JHEP 07 (2000) 045 [hep-th/0007104] [INSPIRE].

[23] D. Chicherin and E. Sokatchev, $N=4$ super-Yang-Mills in LHC superspace part I: classical and quantum theory, JHEP 02 (2017) 062 [arXiv: 1601.06803] [INSPIRE].

[24] L.J. Mason, Twistor actions for non-self-dual fields: a derivation of twistor-string theory, JHEP 10 (2005) 009 [hep-th/0507269] [INSPIRE].

[25] R. Boels, L.J. Mason and D. Skinner, Supersymmetric gauge theories in twistor space, JHEP 02 (2007) 014 [hep-th/0604040] [INSPIRE].

[26] A.A. Rosly and K.G. Selivanov, On amplitudes in selfdual sector of Yang-Mills theory, Phys. Lett. B 399 (1997) 135 [hep-th/9611101] [INSPIRE].

[27] M. Bullimore, L.J. Mason and D. Skinner, MHV diagrams in momentum twistor space, JHEP 12 (2010) 032 [arXiv: 1009.1854] [INSPIRE].

[28] D. Chicherin et al., Correlation functions of the chiral stress-tensor multiplet in $N=4 S Y M$, JHEP 06 (2015) 198 [arXiv:1412.8718] [INSPIRE].

[29] D. Chicherin and E. Sokatchev, $N=4$ super-Yang-Mills in LHC superspace part II: non-chiral correlation functions of the stress-tensor multiplet, JHEP 03 (2017) 048 [arXiv: 1601.06804] [INSPIRE].

[30] D. Chicherin and E. Sokatchev, Composite operators and form factors in $N=4 S Y M$, J. Phys. A 50 (2017) 275402 [arXiv:1605.01386] [INSPIRE]. 
[31] J.M. Drummond, J. Henn, G.P. Korchemsky and E. Sokatchev, Dual superconformal symmetry of scattering amplitudes in $N=4$ super-Yang-Mills theory,

Nucl. Phys. B 828 (2010) 317 [arXiv:0807.1095] [INSPIRE].

[32] M.F. Sohnius, Bianchi identities for supersymmetric gauge theories, Nucl. Phys. B 136 (1978) 461 [inSPIRE].

[33] E. Sokatchev, An action for $N=4$ supersymmetric selfdual Yang-Mills theory, Phys. Rev. D 53 (1996) 2062 [hep-th/9509099] [INSPIRE].

[34] B.M. Zupnik, The action of the supersymmetric $N=2$ gauge theory in harmonic superspace, Phys. Lett. B 183 (1987) 175 [INSPIRE].

[35] N. Berkovits and J. Maldacena, Fermionic T-duality, dual superconformal symmetry and the amplitude/Wilson loop connection, JHEP 09 (2008) 062 [arXiv:0807.3196] [INSPIRE].

[36] N. Beisert, R. Ricci, A.A. Tseytlin and M. Wolf, Dual superconformal symmetry from $A d S_{5} \times S^{5}$ superstring integrability, Phys. Rev. D 78 (2008) 126004 [arXiv:0807.3228] [INSPIRE].

[37] J.M. Drummond, J.M. Henn and J. Plefka, Yangian symmetry of scattering amplitudes in $N=4$ super Yang-Mills theory, JHEP 05 (2009) 046 [arXiv:0902 .2987] [INSPIRE].

[38] A. Galperin, E. Ivanov, S. Kalitsyn, V. Ogievetsky and E. Sokatchev, Unconstrained $N=2$ matter, Yang-Mills and supergravity theories in harmonic superspace, Class. Quant. Grav. 1 (1984) 469 [Erratum ibid. 2 (1985) 127] [INSPIRE].

[39] R.S. Ward, On selfdual gauge fields, Phys. Lett. A 61 (1977) 81 [inSPIRE].

[40] A.S. Galperin, E.A. Ivanov, V.I. Ogievetsky and E. Sokatchev, Gauge field geometry from complex and harmonic analyticities. Kähler and selfdual Yang-Mills cases, Annals Phys. 185 (1988) 1 [INSPIRE].

[41] C. Lovelace, Twistors versus harmonics, arXiv:1006. 4289 [INSPIRE].

[42] M. Bullimore and D. Skinner, Holomorphic linking, loop equations and scattering amplitudes in twistor space, arXiv:1101.1329 [INSPIRE].

[43] F. Cachazo, P. Svrček and E. Witten, MHV vertices and tree amplitudes in gauge theory, JHEP 09 (2004) 006 [hep-th/0403047] [INSPIRE]. 